Supporting Information to the Manuscript:

Syntheses and Characterization of Dicarbonyl-Nitrosyl Complexes of Technetium(I) and Rhenium(I) in Aqueous Media: Spectroscopic, Structural and DFT Analyses

Roger Schibli, Niklaus Marti, Patrick Maurer, Bernhard Spingler, Marie-Line Lehaire, Volker Gramlich, and Charles L. Barnes 


\section{Table of Content:}

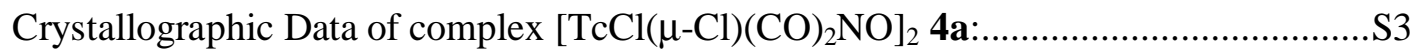

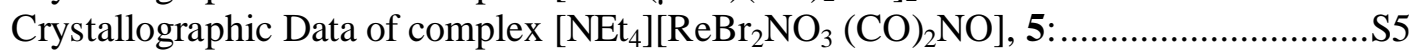

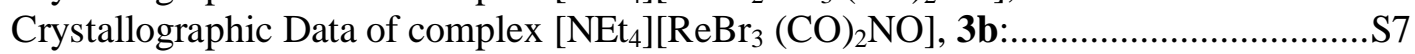

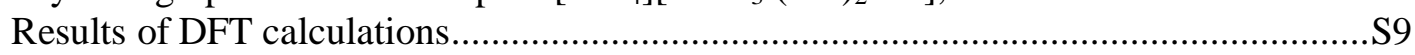

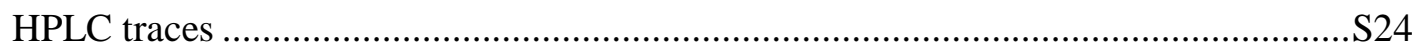

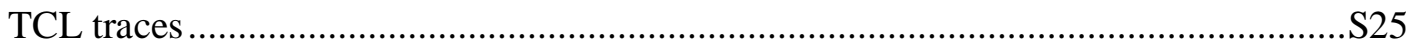


Crystallographic Data of complex $\left[\mathrm{TcCl}(\mu-\mathrm{Cl})(\mathrm{CO})_{2} \mathrm{NO}\right]_{2} 4 \mathrm{a}$ :

Figure 1: ORTEP of complex 4a

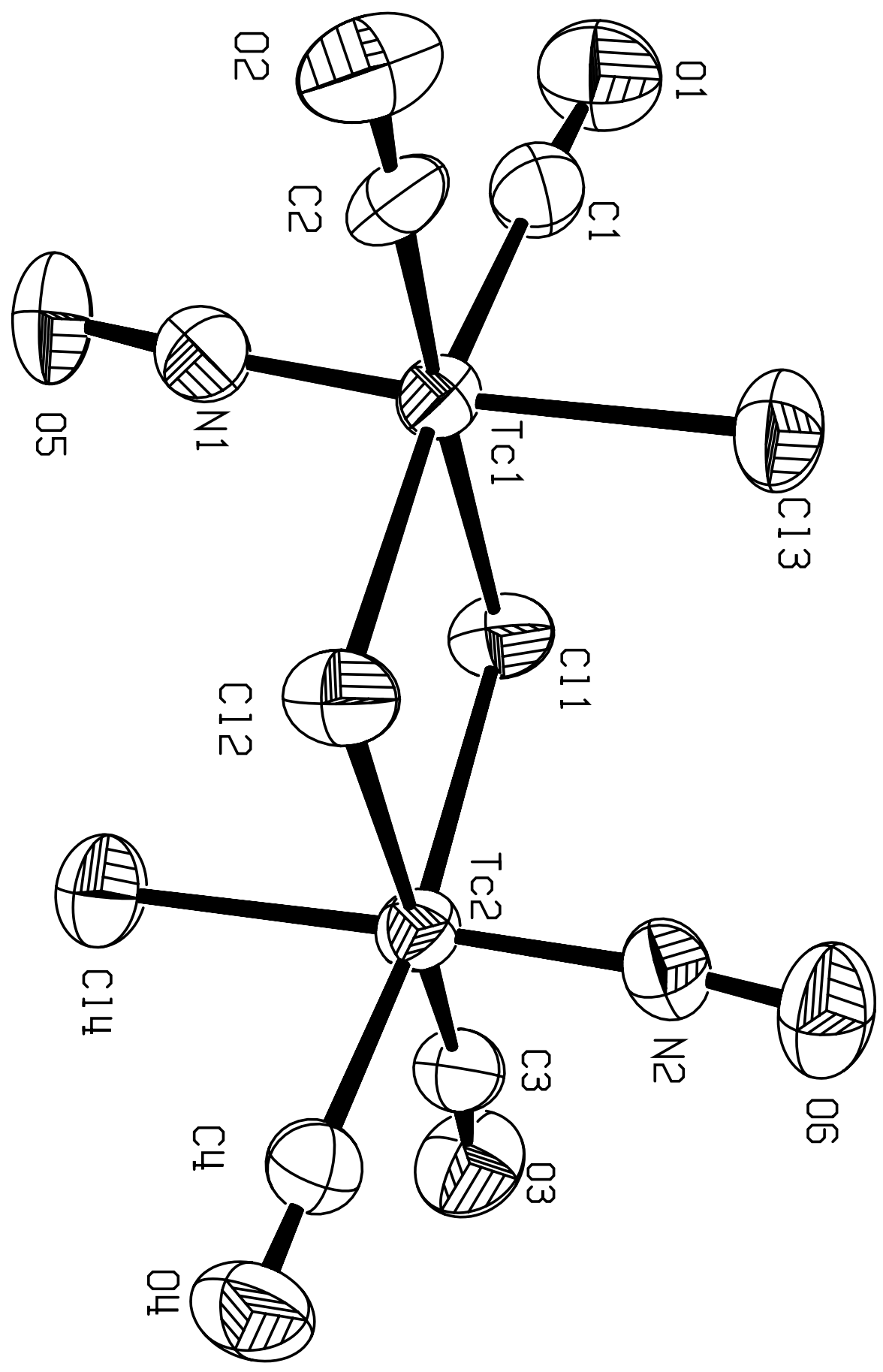


Figure 2: Unit cell content of complex $4 \mathbf{a}$

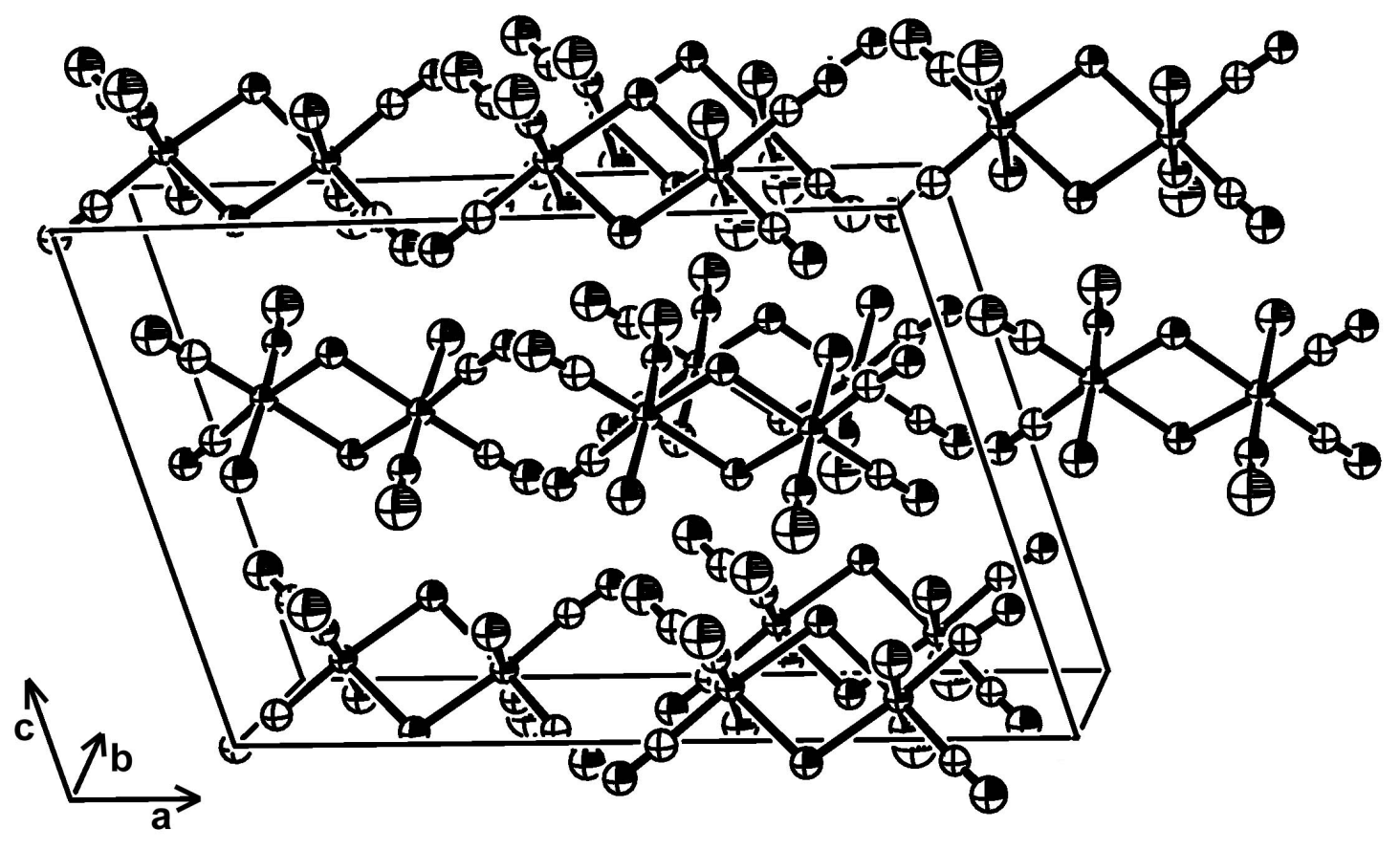


Crystallographic Data of complex $\left[\mathrm{NEt}_{4}\right]\left[\mathrm{ReBr}_{2} \mathrm{NO}_{3}(\mathrm{CO})_{2} \mathrm{NO}\right], 5$ :

Figure 3: ORTEP of complex 5

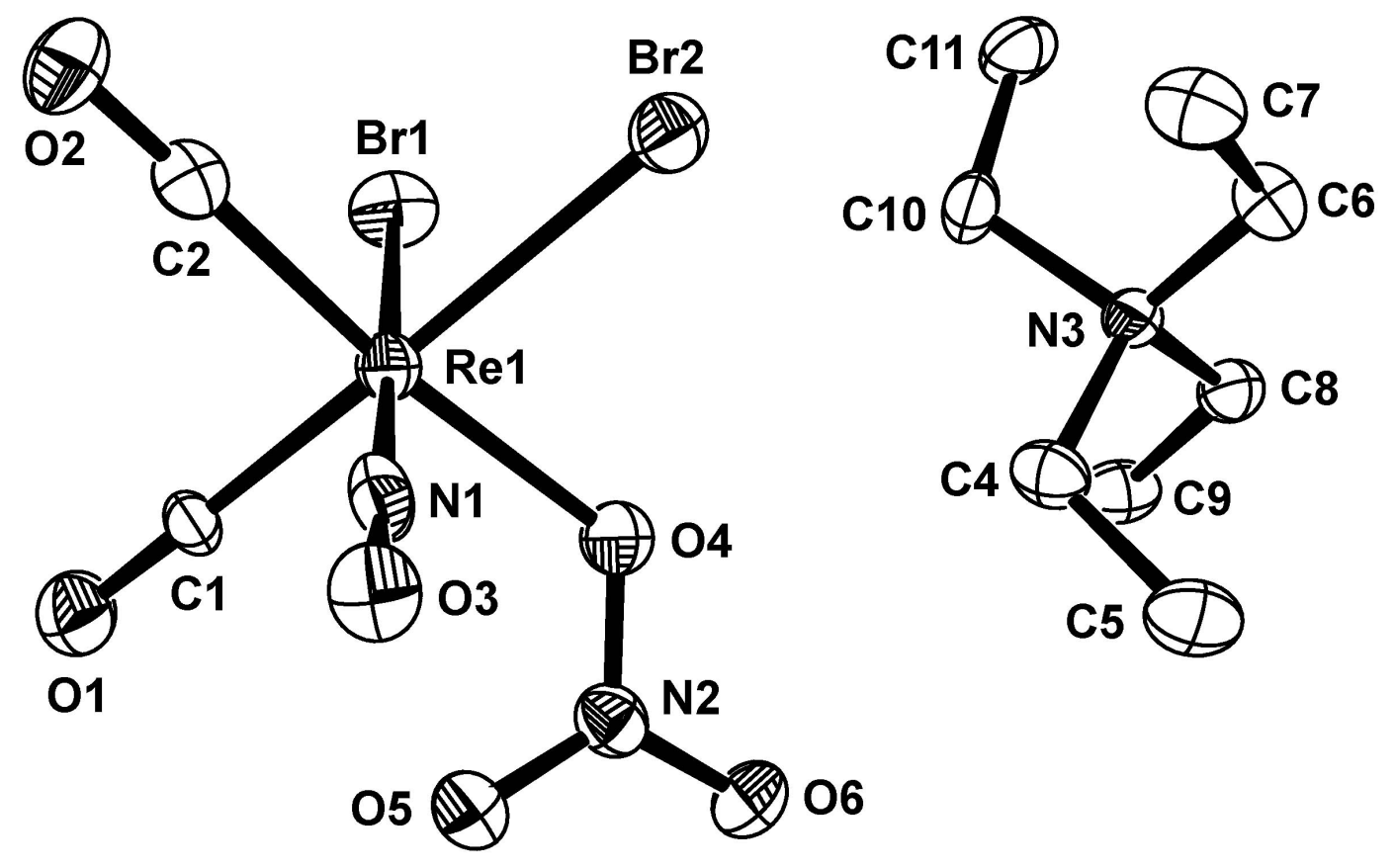


Figure 4: Unit cell content of complex 5

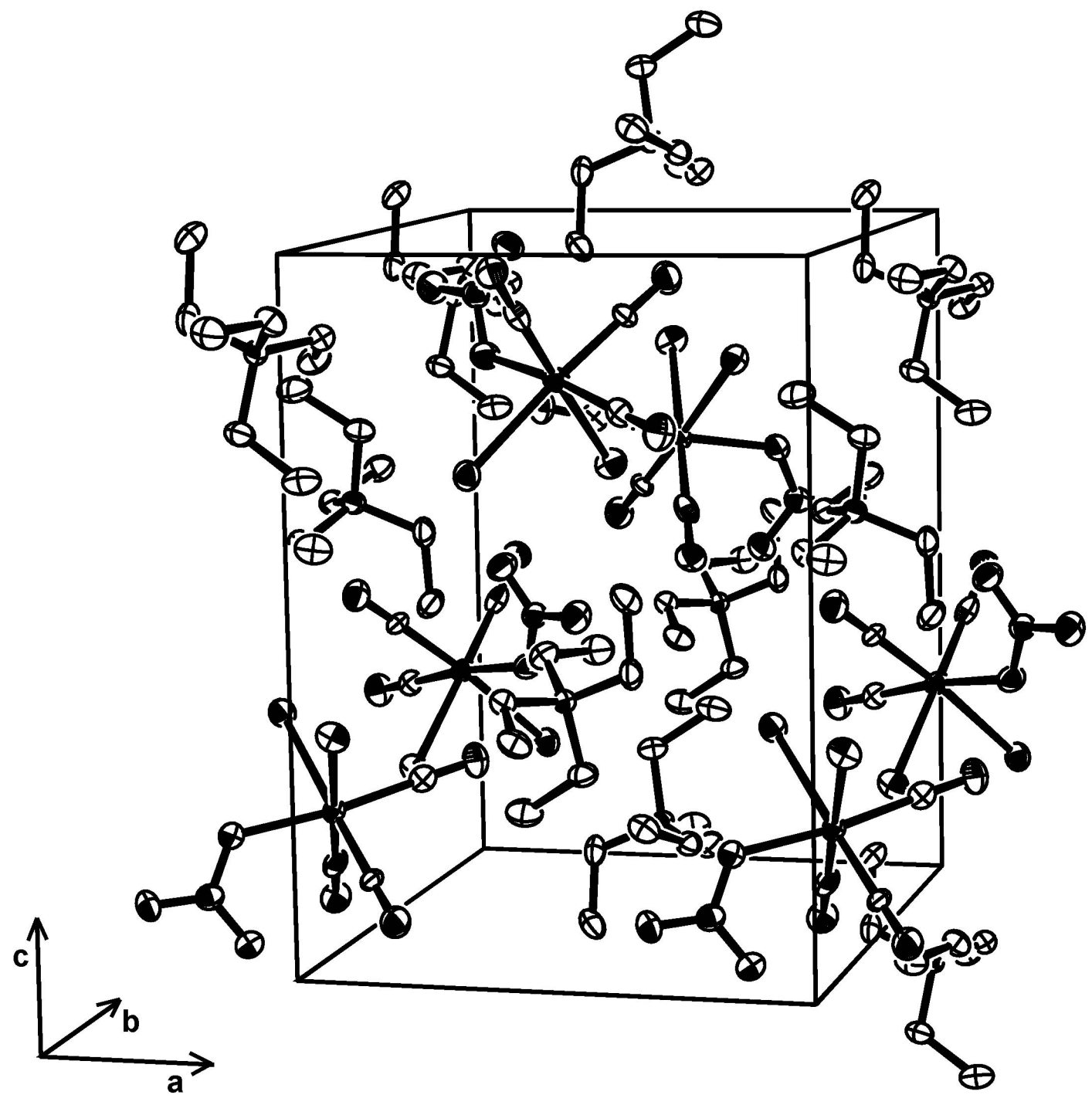




\section{Crystallographic Data of complex $\left[\mathrm{NEt}_{4}\right]\left[\mathrm{ReBr}_{3}(\mathrm{CO})_{2} \mathrm{NO}\right], 3 \mathrm{~b}$ :}

Figure 5: ORTEP of complex $\mathbf{3 b}$
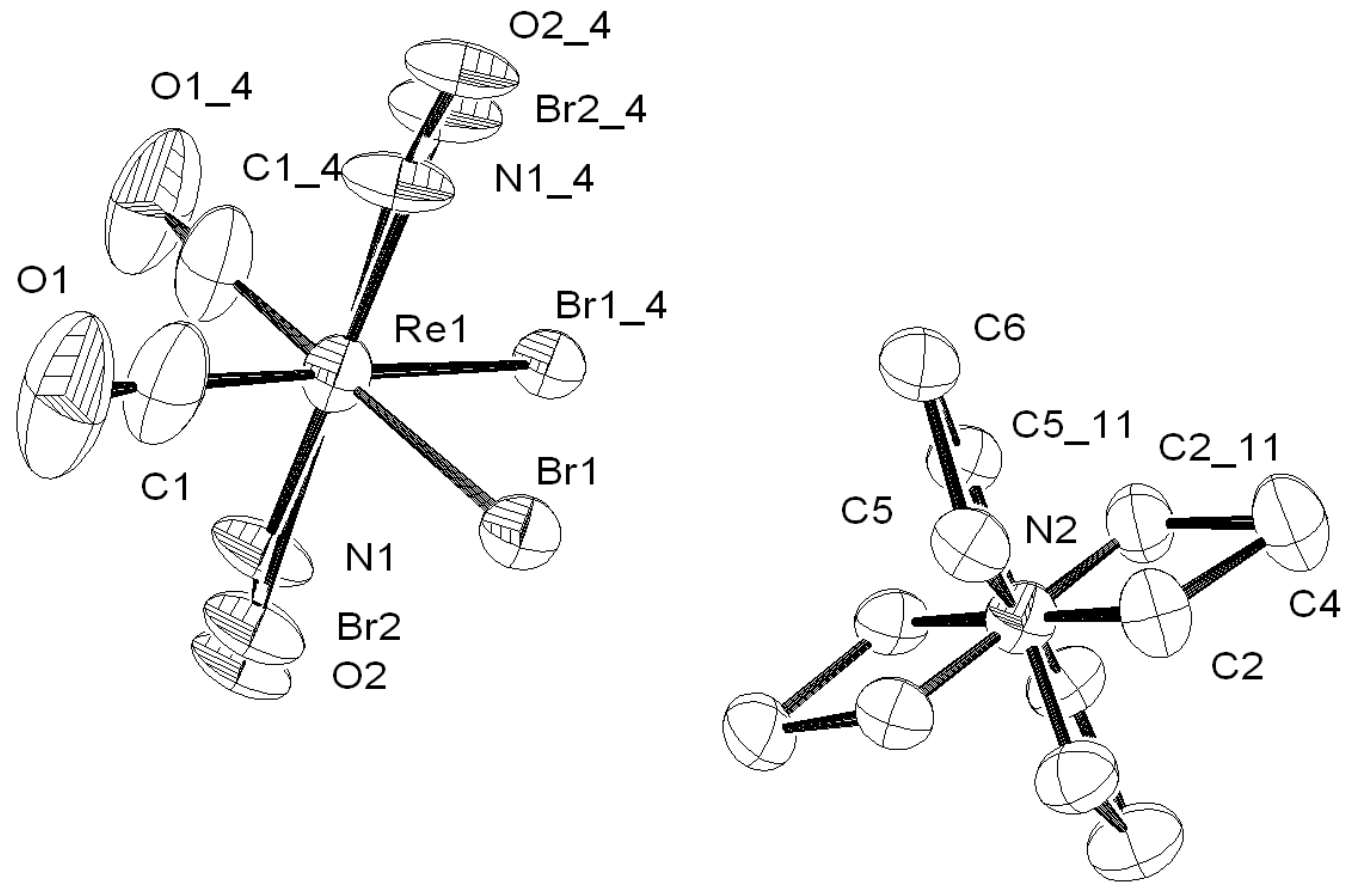

Empirical formula

Formula weight

Temperature

Wavelength

Crystal system

Space group

Unit cell dimensions

Volume

$\mathrm{Z}$

Density (calculated)

Absorption coefficient

$\mathrm{F}(000)$

Crystal size
C10 H20 Br3 N2 O3 Re

642.21

183(2) K

$0.71073 \AA$

Orthorhombic

$\mathrm{Cmcm}$

$\mathrm{a}=8.2101(6) \AA$

$\alpha=90^{\circ}$.

$\mathrm{b}=25.1002(12) \AA$

$\beta=90^{\circ}$.

$\mathrm{c}=8.8348(7) \AA$

$\gamma=90^{\circ}$.

1820.6(2) $\AA^{3}$

4

$2.343 \mathrm{Mg} / \mathrm{m}^{3}$

$13.262 \mathrm{~mm}^{-1}$

1192

$0.37 \times 0.34 \times 0.03 \mathrm{~mm}^{3}$ 
Theta range for data collection

Index ranges

Reflections collected

Independent reflections

Completeness to theta $=25.85^{\circ}$

Absorption correction

Max. and min. transmission

Refinement method

Data / restraints / parameters

Goodness-of-fit on $\mathrm{F}^{2}$

Final $R$ indices [I $>2 \operatorname{sigma}(\mathrm{I})]$

$\mathrm{R}$ indices (all data)

Largest diff. peak and hole
2.61 to $25.85^{\circ}$.

$-10<=\mathrm{h}<=10,-29<=\mathrm{k}<=29,-10<=\mathrm{l}<=10$

6130

$978[\mathrm{R}(\mathrm{int})=0.1083]$

$96.8 \%$

Numerical

0.4646 and 0.0451

Full-matrix least-squares on $\mathrm{F}^{2}$

$978 / 22 / 75$

1.114

$\mathrm{R} 1=0.0682, \mathrm{wR} 2=0.1927$

$\mathrm{R} 1=0.0729, \mathrm{wR} 2=0.1971$

5.408 and -4.212 e. $\AA^{-3}$

\section{Note:}

Atoms N1, $\mathrm{O} 2$ and $\mathrm{Br} 2$ as well as $\mathrm{C} 2$ and $\mathrm{C} 5$ reveal a $50 \%$ displacement. 


\section{Results of DFT calculations}

The numbering schemes of each isomer of compound $\mathbf{3}$.

\section{Compound 3b}
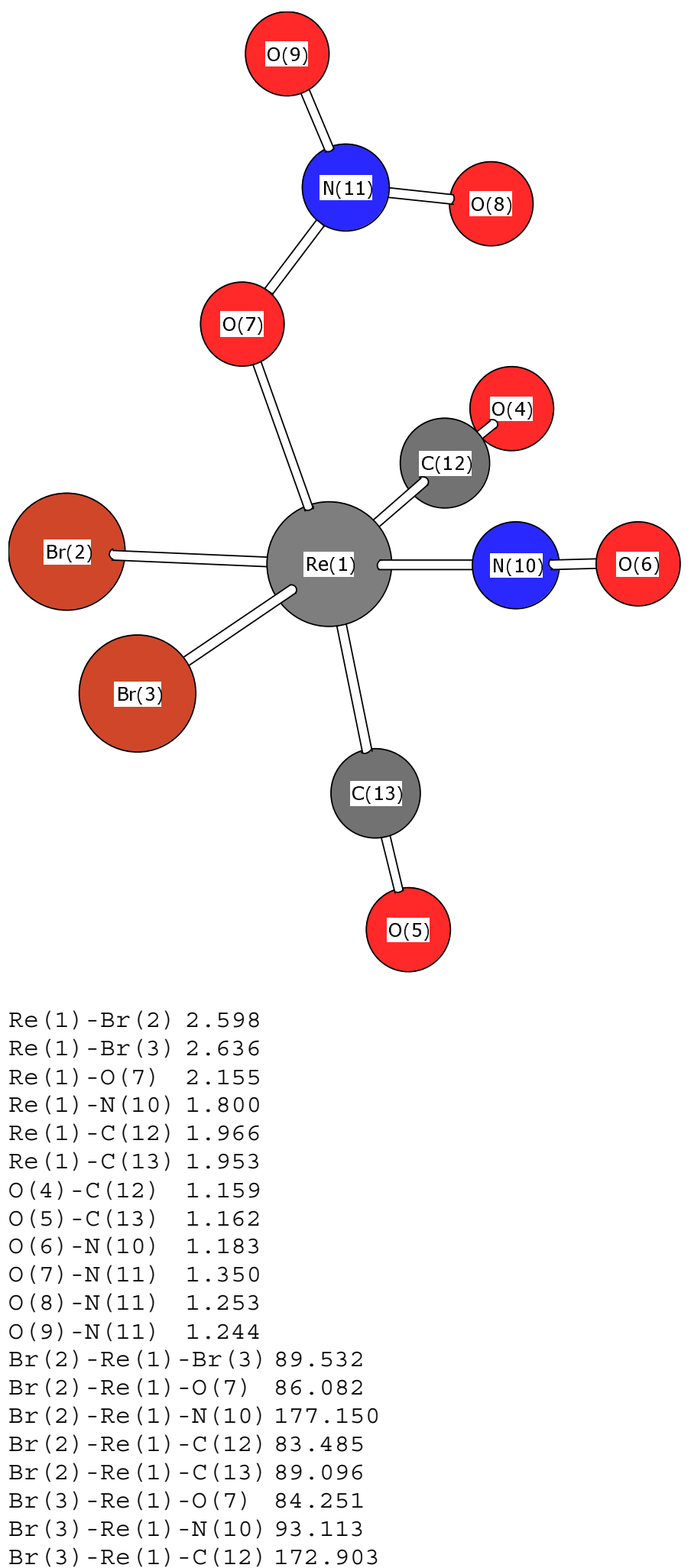


$\begin{array}{ll}\operatorname{Br}(3)-\operatorname{Re}(1)-\mathrm{C}(13) & 89.413 \\ \mathrm{O}(7)-\operatorname{Re}(1)-\mathrm{N}(10) & 93.106 \\ \mathrm{O}(7)-\operatorname{Re}(1)-\mathrm{C}(12) & 94.004 \\ \mathrm{O}(7)-\operatorname{Re}(1)-\mathrm{C}(13) & 172.062 \\ \mathrm{~N}(10)-\operatorname{Re}(1)-\mathrm{C}(12) & 93.847 \\ \mathrm{~N}(10)-\operatorname{Re}(1)-\mathrm{C}(13) & 92.008 \\ \mathrm{C}(12)-\operatorname{Re}(1)-\mathrm{C}(13) & 91.703 \\ \operatorname{Re}(1)-\mathrm{O}(7)-\mathrm{N}(11) & 120.122 \\ \operatorname{Re}(1)-\mathrm{N}(10)-\mathrm{O}(6) & 179.051 \\ \mathrm{O}(7)-\mathrm{N}(11)-\mathrm{O}(8) & 118.840 \\ \mathrm{O}(7)-\mathrm{N}(11)-\mathrm{O}(9) & 116.464 \\ \mathrm{O}(8)-\mathrm{N}(11)-\mathrm{O}(9) & 124.693 \\ \operatorname{Re}(1)-\mathrm{C}(12)-\mathrm{O}(4) & 177.082 \\ \operatorname{Re}(1)-\mathrm{C}(13)-\mathrm{O}(5) & 177.351\end{array}$

\section{Isomer 1}
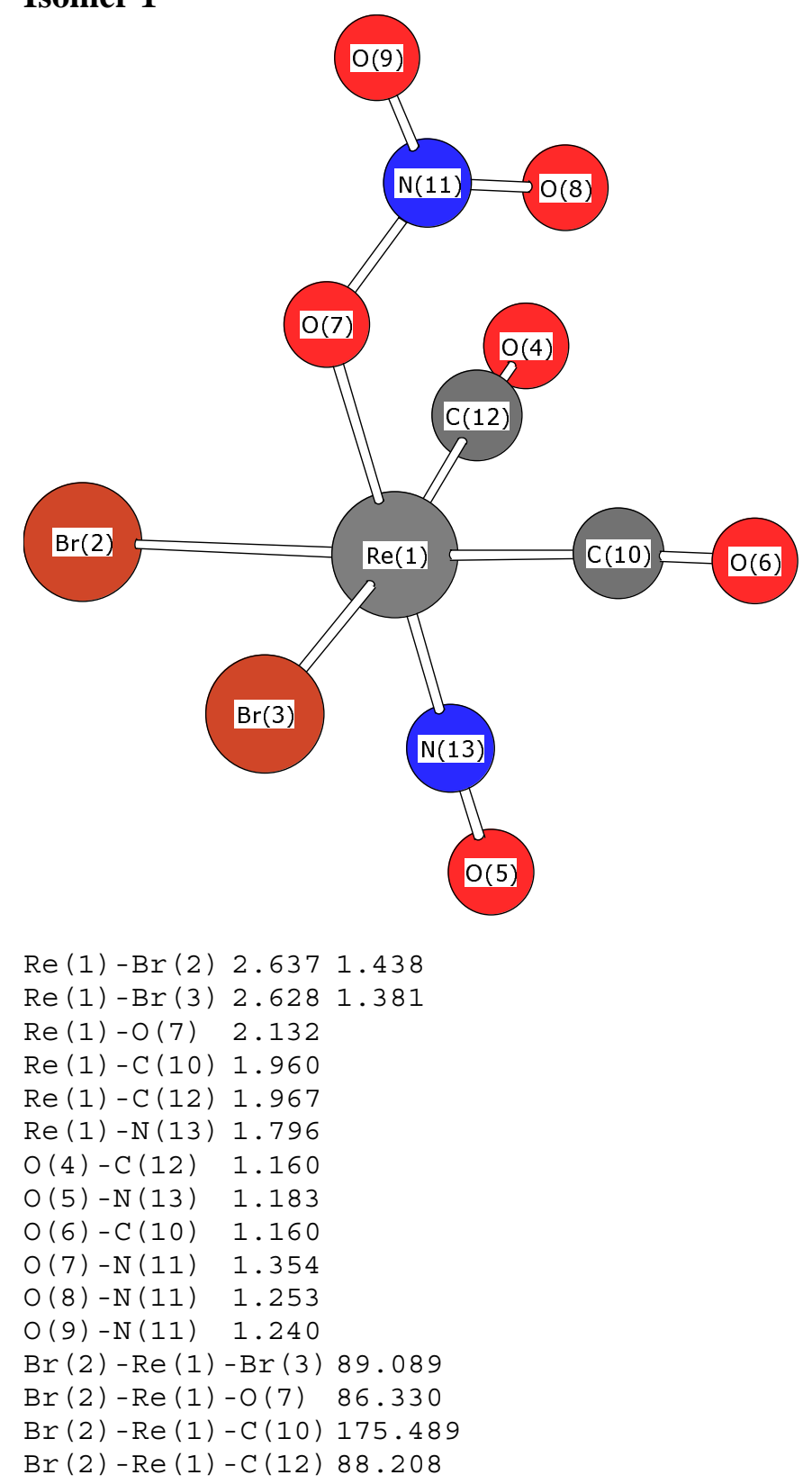


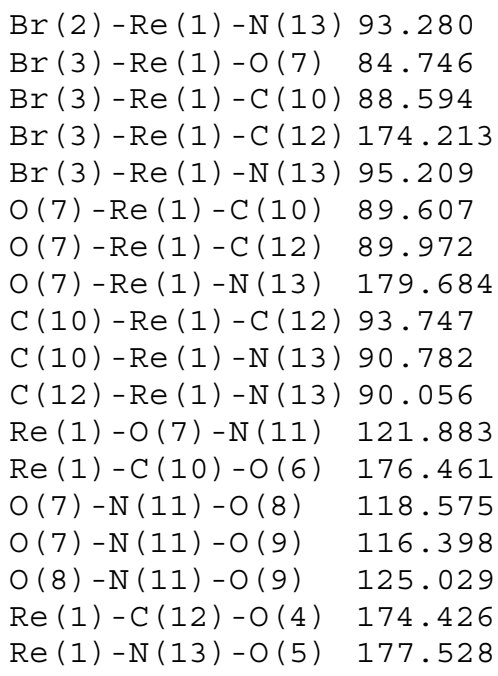

\section{Compound $4 a / b$}

The numbering schemes of each isomer is shown for the compounds with $\mathrm{M}=\mathrm{Tc}, \mathrm{X}=\mathrm{Cl}$. The numbering scheme is identical for the corresponding isomer with $\mathrm{M}=\mathrm{Re}, \mathrm{X}=\mathrm{Br}$.
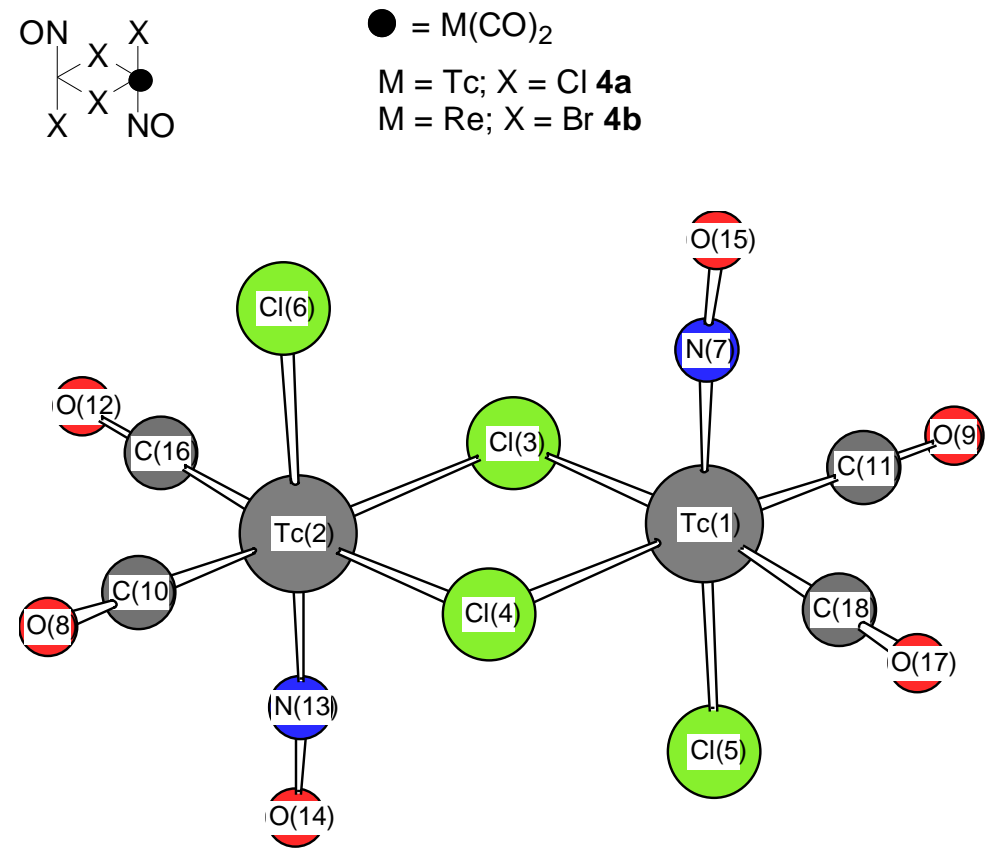

Bond lengths in $\AA$

\begin{tabular}{|c|c|}
\hline $\mathrm{M}=\mathrm{TC} ; \quad \mathrm{X}=\mathrm{Cl}$ & $\mathrm{M}=\mathrm{Re} \quad ; \mathrm{X}=\mathrm{Br}$ \\
\hline $\mathrm{TC}(1)-\mathrm{Cl}(3) 2.520$ & $\operatorname{Re}(1)-\operatorname{Br}(3) 2.691$ \\
\hline $\mathrm{TC}(1)-\mathrm{Cl}(4) 2.517$ & $\operatorname{Re}(1)-\operatorname{Br}(4) 2.688$ \\
\hline $\mathrm{TC}(1)-\mathrm{Cl}(5) 2.397$ & $\operatorname{Re}(1)-\operatorname{Br}(5) 2.567$ \\
\hline $\mathrm{TC}(1)-\mathrm{N}(7) \quad 1.812$ & $\operatorname{Re}(1)-\mathrm{N}(7) \quad 1.810$ \\
\hline $\mathrm{TC}(1)-\mathrm{C}(11) 1.972$ & $\operatorname{Re}(1)-C(11) 1.954$ \\
\hline $\operatorname{TC}(1)-\mathrm{C}(18) 1.975$ & $\operatorname{Re}(1)-C(18) 1.954$ \\
\hline $\mathrm{TC}(2)-\mathrm{Cl}(3) 2.523$ & $\operatorname{Re}(2)-\operatorname{Br}(3) 2.688$ \\
\hline $\mathrm{TC}(2)-\mathrm{Cl}(4) 2.532$ & $\operatorname{Re}(2)-\operatorname{Br}(4) 2.696$ \\
\hline $\mathrm{TC}(2)-\mathrm{Cl}(6) 2.387$ & $\operatorname{Re}(2)-\operatorname{Br}(6) 2.561$ \\
\hline
\end{tabular}




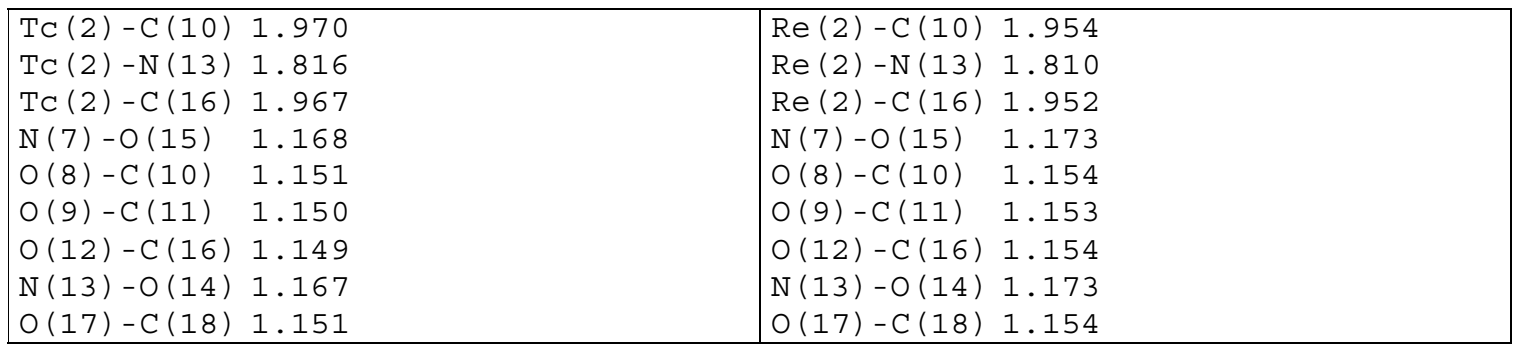

Bond angles in degrees

\begin{tabular}{|c|c|}
\hline $\mathrm{M}=\mathrm{TC} ; \quad \mathrm{X}=\mathrm{Cl}$ & $\mathrm{M}=\mathrm{Re} \quad ; \mathrm{X}=\mathrm{Br}$ \\
\hline $\mathrm{Cl}(3)-\mathrm{TC}(1)-\mathrm{Cl}(4) 83.011$ & $\operatorname{Br}(3)-\operatorname{Re}(1)-\operatorname{Br}(4) 84.108$ \\
\hline $\mathrm{Cl}(3)-\mathrm{TC}(1)-\mathrm{Cl}(5) 89.528$ & $\operatorname{Br}(3)-\operatorname{Re}(1)-\operatorname{Br}(5) 89.820$ \\
\hline $\mathrm{Cl}(3)-\mathrm{TC}(1)-\mathrm{N}(7) \quad 92.782$ & $\operatorname{Br}(3)-\operatorname{Re}(1)-\mathrm{N}(7) \quad 92.217$ \\
\hline $\mathrm{Cl}(3)-\mathrm{TC}(1)-\mathrm{C}(11) 92.773$ & $\operatorname{Br}(3)-\operatorname{Re}(1)-C(11) 92.803$ \\
\hline $\mathrm{Cl}(3)-\mathrm{TC}(1)-\mathrm{C}(18) 172.580$ & $\operatorname{Br}(3)-\operatorname{Re}(1)-C(18) 173.335$ \\
\hline $\mathrm{Cl}(4)-\mathrm{TC}(1)-\mathrm{Cl}(5) 88.841$ & $\operatorname{Br}(4)-\operatorname{Re}(1)-\operatorname{Br}(5) 89.456$ \\
\hline $\mathrm{Cl}(4)-\mathrm{TC}(1)-\mathrm{N}(7) \quad 93.739$ & $\operatorname{Br}(4)-\operatorname{Re}(1)-\mathrm{N}(7) \quad 93.027$ \\
\hline $\mathrm{Cl}(4)-\mathrm{TC}(1)-\mathrm{C}(11) 172.358$ & $\operatorname{Br}(4)-\operatorname{Re}(1)-C(11) 173.762$ \\
\hline $\mathrm{Cl}(4)-\mathrm{TC}(1)-\mathrm{C}(18) 91.869$ & $\operatorname{Br}(4)-\operatorname{Re}(1)-C(18) 91.296$ \\
\hline $\mathrm{Cl}(5)-\mathrm{TC}(1)-\mathrm{N}(7) \quad 176.726$ & $\operatorname{Br}(5)-\operatorname{Re}(1)-N(7) \quad 176.930$ \\
\hline $\mathrm{Cl}(5)-\mathrm{TC}(1)-\mathrm{C}(11) 84.741$ & $\operatorname{Br}(5)-\operatorname{Re}(1)-C(11) 85.111$ \\
\hline $\mathrm{Cl}(5)-\mathrm{TC}(1)-\mathrm{C}(18) 84.985$ & $\operatorname{Br}(5)-\operatorname{Re}(1)-C(18) 85.293$ \\
\hline $\mathrm{N}(7)-\mathrm{TC}(1)-\mathrm{C}(11) \quad 92.828$ & $N(7)-\operatorname{Re}(1)-C(11) \quad 92.501$ \\
\hline $\mathrm{N}(7)-\mathrm{TC}(1)-\mathrm{C}(18) \quad 92.912$ & $N(7)-\operatorname{Re}(1)-C(18)$ \\
\hline $\mathrm{C}(11)-\mathrm{TC}(1)-\mathrm{C}(18) 91.696$ & $C(11)-\operatorname{Re}(1)-C(18) 91.305$ \\
\hline $\mathrm{Cl}(3)-\mathrm{TC}(2)-\mathrm{Cl}(4) 82.630$ & $\operatorname{Br}(3)-\operatorname{Re}(2)-\operatorname{Br}(4) 84.020$ \\
\hline $\mathrm{Cl}(3)-\mathrm{TC}(2)-\mathrm{Cl}(6) 89.121$ & $\operatorname{Br}(3)-\operatorname{Re}(2)-\operatorname{Br}(6) 89.421$ \\
\hline $\mathrm{Cl}(3)-\mathrm{TC}(2)-\mathrm{C}(10) 172.464$ & $\operatorname{Br}(3)-\operatorname{Re}(2)-C(10) 173.335$ \\
\hline $\mathrm{Cl}(3)-\mathrm{TC}(2)-\mathrm{N}(13) 93.032$ & $\operatorname{Br}(3)-\operatorname{Re}(2)-\mathrm{N}(13) 92.532$ \\
\hline $\mathrm{Cl}(3)-\mathrm{TC}(2)-\mathrm{C}(16) 92.845$ & $\operatorname{Br}(3)-\operatorname{Re}(2)-C(16) 93.364$ \\
\hline $\mathrm{Cl}(4)-\mathrm{TC}(2)-\mathrm{Cl}(6) 88.794$ & $\operatorname{Br}(4)-\operatorname{Re}(2)-\operatorname{Br}(6) 89.413$ \\
\hline $\mathrm{Cl}(4)-\mathrm{TC}(2)-\mathrm{C}(10) 92.682$ & $\operatorname{Br}(4)-\operatorname{Re}(2)-C(10) 92.144$ \\
\hline $\mathrm{Cl}(4)-\mathrm{TC}(2)-\mathrm{N}(13) 92.064$ & $\operatorname{Br}(4)-\operatorname{Re}(2)-N(13) 91.256$ \\
\hline $\mathrm{Cl}(4)-\mathrm{TC}(2)-\mathrm{C}(16) 173.793$ & $\operatorname{Br}(4)-\operatorname{Re}(2)-C(16) 175.512$ \\
\hline $\mathrm{Cl}(6)-\mathrm{TC}(2)-\mathrm{C}(10) 84.867$ & $\operatorname{Br}(6)-\operatorname{Re}(2)-C(10) 85.078$ \\
\hline $\mathrm{Cl}(6)-\mathrm{TC}(2)-\mathrm{N}(13) 177.762$ & $\operatorname{Br}(6)-\operatorname{Re}(2)-\mathrm{N}(13) 177.998$ \\
\hline $\mathrm{Cl}(6)-\mathrm{TC}(2)-\mathrm{C}(16) 86.879$ & $\operatorname{Br}(6)-\operatorname{Re}(2)-C(16) 86.907$ \\
\hline $\mathrm{C}(10)-\mathrm{TC}(2)-\mathrm{N}(13) 93.034$ & $C(10)-\operatorname{Re}(2)-N(13) 93.002$ \\
\hline $\mathrm{C}(10)-\mathrm{TC}(2)-\mathrm{C}(16) 91.364$ & $C(10)-\operatorname{Re}(2)-C(16) 90.105$ \\
\hline $\mathrm{N}(13)-\mathrm{TC}(2)-\mathrm{C}(16) 92.424$ & $N(13)-\operatorname{Re}(2)-C(16) 92.509$ \\
\hline $\mathrm{TC}(1)-\mathrm{Cl}(3)-\mathrm{TC}(2) 97.253$ & $\operatorname{Re}(1)-\operatorname{Br}(3)-\operatorname{Re}(2) 96.001$ \\
\hline $\mathrm{TC}(1)-\mathrm{Cl}(4)-\mathrm{TC}(2) 97.096$ & $\operatorname{Re}(1)-\operatorname{Br}(4)-\operatorname{Re}(2) 95.868$ \\
\hline $\mathrm{TC}(1)-\mathrm{N}(7)-\mathrm{O}(15) \quad 175.568$ & $\operatorname{Re}(1)-N(7)-O(15) \quad 176.364$ \\
\hline $\mathrm{TC}(2)-\mathrm{C}(10)-\mathrm{O}(8)$ & $\operatorname{Re}(2)-C(10)-O(8)$ \\
\hline $\mathrm{TC}(1)-\mathrm{C}(11)-\mathrm{O}(9) \quad 179.553$ & $\operatorname{Re}(1)-C(11)-O(9) \quad 179.553$ \\
\hline $\mathrm{TC}(2)-\mathrm{N}(13)-\mathrm{O}(14) 176.187$ & $\operatorname{Re}(2)-N(13)-O(14) 176.965$ \\
\hline $\mathrm{TC}(2)-\mathrm{C}(16)-\mathrm{O}(12) 179.553$ & $\operatorname{Re}(2)-C(16)-O(12) 178.126$ \\
\hline $\mathrm{TC}(1)-\mathrm{C}(18)-\mathrm{O}(17) 179.684$ & $\operatorname{Re}(1)-C(18)-O(17) 179.451$ \\
\hline
\end{tabular}

\section{Isomer 1:}

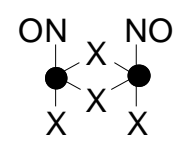




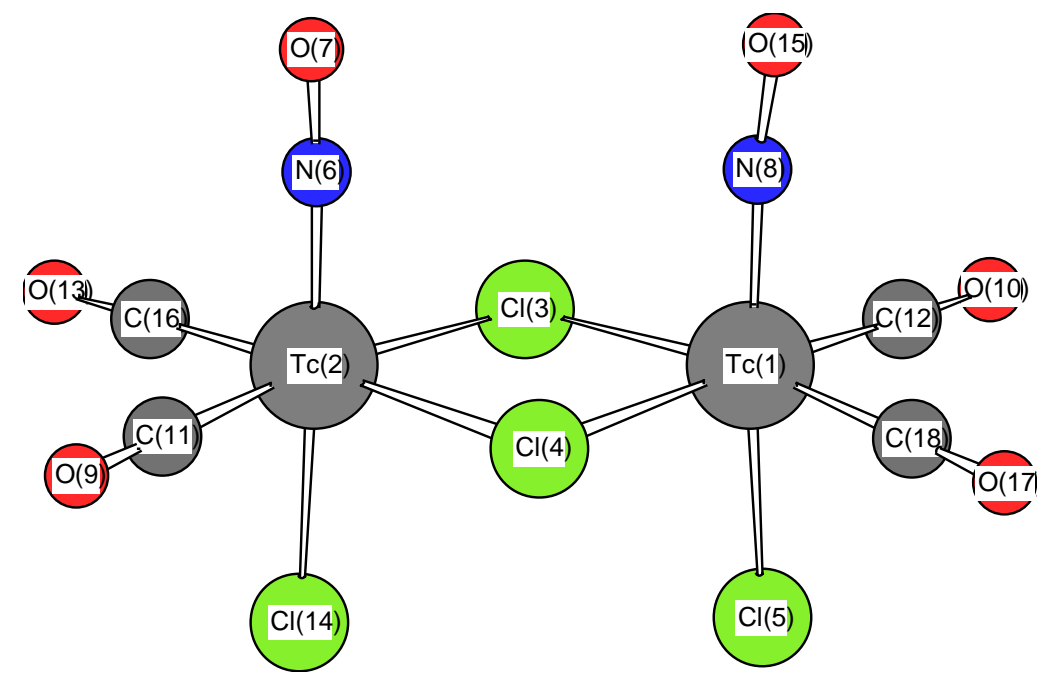

Bond lengths in $\AA$

\begin{tabular}{|c|c|c|c|}
\hline \multicolumn{2}{|l|}{$\mathrm{M}=\mathrm{TC} ; \quad \mathrm{X}=\mathrm{Cl}$} & \multicolumn{2}{|c|}{$\mathrm{M}=\mathrm{Re} \quad ; \mathrm{X}=\mathrm{Br}$} \\
\hline $\operatorname{TC}(1)-\mathrm{Cl}(3) 2$ & 2.528 & $\operatorname{Re}(1)-\operatorname{Br}(3)$ & 2.690 \\
\hline $\operatorname{TC}(1)-\mathrm{Cl}(4) 2$ & 2.524 & $\operatorname{Re}(1)-\operatorname{Br}(4)$ & 2.690 \\
\hline $\mathrm{TC}(1)-\mathrm{Cl}(5) 2$ & 2.382 & $\operatorname{Re}(1)-\operatorname{Br}(5)$ & 2.558 \\
\hline $\mathrm{TC}(1)-\mathrm{N}(8) \quad 1$ & 1.812 & $\operatorname{Re}(1)-\mathrm{N}(8)$ & 1.812 \\
\hline $\operatorname{TC}(1)-\mathrm{C}(12) 1$ & 1.970 & $\operatorname{Re}(1)-C(12)$ & 1.959 \\
\hline $\operatorname{TC}(1)-C(18) 1$ & 1.972 & $\operatorname{Re}(1)-C(18)$ & 1.960 \\
\hline $\operatorname{TC}(2)-\mathrm{Cl}(3) 2$ & 2.527 & $\operatorname{Re}(2)-\operatorname{Br}(3)$ & 2.692 \\
\hline $\operatorname{TC}(2)-\mathrm{Cl}(4) 2$ & 2.531 & $\operatorname{Re}(2)-\operatorname{Br}(4)$ & 2.698 \\
\hline $\mathrm{TC}(2)-\mathrm{N}(6) \quad 1$ & 1.813 & $\operatorname{Re}(2)-\mathrm{N}(6)$ & 1.813 \\
\hline $\operatorname{TC}(2)-C(11) 1$ & 1.970 & $\operatorname{Re}(2)-C(11)$ & 1.958 \\
\hline $\operatorname{TC}(2)-\mathrm{Cl}(14)$ & 2.377 & $\operatorname{Re}(2)-\operatorname{Br}(14$ & 2.554 \\
\hline $\mathrm{TC}(2)-\mathrm{C}(16) 1$ & 1.973 & $\operatorname{Re}(2)-C(16)$ & 1.958 \\
\hline$N(6)-O(7) \quad 1$ & 1.173 & $\mathrm{~N}(6)-\mathrm{O}(7)$ & 1.177 \\
\hline $\mathrm{N}(8)-\mathrm{O}(15)$ & 1.172 & $\mathrm{~N}(8)-\mathrm{O}(15)$ & 1.176 \\
\hline$O(9)-C(11) \quad 1$ & 1.151 & $O(9)-C(11)$ & 1.153 \\
\hline$O(10)-C(12) 1$ & 1.150 & $\mathrm{O}(10)-\mathrm{C}(12)$ & 1.152 \\
\hline$O(13)-C(16) 1$ & 1.150 & $\mathrm{O}(13)-\mathrm{C}(16)$ & 1.153 \\
\hline$O(17)-C(18) 1$ & 1.150 & $\mathrm{O}(17)-\mathrm{C}(18)$ & 1.152 \\
\hline
\end{tabular}

Bond angles in degrees

\begin{tabular}{|c|c|}
\hline $\mathrm{M}=\mathrm{TC} ; \quad \mathrm{X}=\mathrm{Cl}$ & $\mathrm{M}=\mathrm{Re} \quad ; \mathrm{X}=\mathrm{Br}$ \\
\hline $\mathrm{Cl}(3)-\mathrm{TC}(1)-\mathrm{Cl}(4) 82.225$ & $\operatorname{Br}(3)-\operatorname{Re}(1)-\operatorname{Br}(4) 83.092$ \\
\hline $\mathrm{Cl}(3)-\mathrm{TC}(1)-\mathrm{Cl}(5) 89.145$ & $\operatorname{Br}(3)-\operatorname{Re}(1)-\operatorname{Br}(5) 89.432$ \\
\hline $\mathrm{Cl}(3)-\mathrm{TC}(1)-\mathrm{N}(8) \quad 93.539$ & $\operatorname{Br}(3)-\operatorname{Re}(1)-\mathrm{N}(8) \quad 92.628$ \\
\hline $\mathrm{Cl}(3)-\mathrm{TC}(1)-\mathrm{C}(12) 92.803$ & $\operatorname{Br}(3)-\operatorname{Re}(1)-C(12) 92.644$ \\
\hline $\mathrm{Cl}(3)-\mathrm{TC}(1)-\mathrm{C}(18) 171.452$ & $\operatorname{Br}(3)-\operatorname{Re}(1)-C(18) 172.723$ \\
\hline $\mathrm{Cl}(4)-\mathrm{TC}(1)-\mathrm{Cl}(5) 89.860$ & $\operatorname{Br}(4)-\operatorname{Re}(1)-\operatorname{Br}(5) 89.628$ \\
\hline $\mathrm{Cl}(4)-\mathrm{TC}(1)-\mathrm{N}(8) \quad 93.859$ & $\operatorname{Br}(4)-\operatorname{Re}(1)-\mathrm{N}(8) \quad 93.352$ \\
\hline $\mathrm{Cl}(4)-\mathrm{TC}(1)-\mathrm{C}(12) 172.255$ & $\operatorname{Br}(4)-\operatorname{Re}(1)-C(12) 173.349$ \\
\hline $\mathrm{Cl}(4)-\mathrm{TC}(1)-\mathrm{C}(18) 92.015$ & $\operatorname{Br}(4)-\operatorname{Re}(1)-C(18) 91.962$ \\
\hline $\mathrm{Cl}(5)-\mathrm{TC}(1)-\mathrm{N}(8) \quad 175.671$ & $\operatorname{Br}(5)-\operatorname{Re}(1)-N(8) \quad 176.561$ \\
\hline $\mathrm{Cl}(5)-\mathrm{TC}(1)-\mathrm{C}(12) 84.106$ & $\operatorname{Br}(5)-\operatorname{Re}(1)-C(12) 85.209$ \\
\hline $\mathrm{Cl}(5)-\mathrm{TC}(1)-\mathrm{C}(18) 84.512$ & $\operatorname{Br}(5)-\operatorname{Re}(1)-C(18) 85.185$ \\
\hline $\mathrm{N}(8)-\mathrm{TC}(1)-\mathrm{C}(12) \quad 92.370$ & $N(8)-\operatorname{Re}(1)-C(12) \quad 91.941$ \\
\hline$N(8)-T C(1)-C(18) \quad 93.146$ & $N(8)-\operatorname{Re}(1)-C(18)$ \\
\hline $\mathrm{C}(12)-\mathrm{TC}(1)-\mathrm{C}(18) 92.238$ & $C(12)-\operatorname{Re}(1)-C(18) 91.784$ \\
\hline $\mathrm{Cl}(3)-\mathrm{TC}(2)-\mathrm{Cl}(4) 82.113$ & $\operatorname{Br}(3)-\operatorname{Re}(2)-\operatorname{Br}(4) 82.913$ \\
\hline $\mathrm{Cl}(3)-\mathrm{TC}(2)-\mathrm{N}(6) \quad 93.146$ & $\operatorname{Br}(3)-\operatorname{Re}(2)-\mathrm{N}(6) \quad 92.824$ \\
\hline
\end{tabular}




\begin{tabular}{|c|c|}
\hline $\mathrm{Cl}(3)-\mathrm{TC}(2)-\mathrm{C}(11) 172.365$ & $\operatorname{Br}(3)-\operatorname{Re}(2)-C(11) 173.157$ \\
\hline $\mathrm{Cl}(3)-\mathrm{TC}(2)-\mathrm{Cl}(14) \quad 90.210$ & $\operatorname{Br}(3)-\operatorname{Re}(2)-\operatorname{Br}(14) \quad 89.658$ \\
\hline $\mathrm{Cl}(3)-\mathrm{TC}(2)-\mathrm{C}(16) 92.634$ & $\operatorname{Br}(3)-\operatorname{Re}(2)-C(16) 92.286$ \\
\hline $\mathrm{Cl}(4)-\mathrm{TC}(2)-\mathrm{N}(6) \quad 91.847$ & $\operatorname{Br}(4)-\operatorname{Re}(2)-N(6) \quad 91.215$ \\
\hline $\mathrm{Cl}(4)-\mathrm{TC}(2)-\mathrm{C}(11) 92.590$ & $\operatorname{Br}(4)-\operatorname{Re}(2)-C(11) 92.382$ \\
\hline $\mathrm{Cl}(4)-\mathrm{TC}(2)-\mathrm{Cl}(14) \quad 88.133$ & $\operatorname{Br}(4)-\operatorname{Re}(2)-\operatorname{Br}(14) \quad 88.621$ \\
\hline $\mathrm{Cl}(4)-\mathrm{TC}(2)-\mathrm{C}(16) 172.345$ & $\operatorname{Br}(4)-\operatorname{Re}(2)-C(16) 173.157$ \\
\hline $\mathrm{N}(6)-\mathrm{TC}(2)-\mathrm{C}(11) \quad 92.513$ & $\mathrm{~N}(6)-\operatorname{Re}(2)-\mathrm{C}(11) \quad 92.245$ \\
\hline $\mathrm{N}(6)-\mathrm{TC}(2)-\mathrm{Cl}(14) 176.605$ & $\mathrm{~N}(6)-\operatorname{Re}(2)-\operatorname{Br}(14) 177.488$ \\
\hline $\mathrm{N}(6)-\mathrm{TC}(2)-\mathrm{C}(16) \quad 94.004$ & $N(6)-\operatorname{Re}(2)-C(16) \quad 93.901$ \\
\hline $\mathrm{C}(11)-\mathrm{TC}(2)-\mathrm{Cl}(14) \quad 84.101$ & $C(11)-\operatorname{Re}(2)-\operatorname{Br}(14) \quad 85.246$ \\
\hline $\mathrm{C}(11)-\mathrm{TC}(2)-\mathrm{C}(16) 92.077$ & $C(11)-\operatorname{Re}(2)-C(16) 91.959$ \\
\hline $\mathrm{Cl}(14)-\mathrm{TC}(2)-\mathrm{C}(16) \quad 86.316$ & $\operatorname{Br}(14)-\operatorname{Re}(2)-\mathrm{C}(16) \quad 86.466$ \\
\hline $\mathrm{TC}(1)-\mathrm{Cl}(3)-\mathrm{TC}(2) 97.568$ & $\operatorname{Re}(1)-\operatorname{Br}(3)-\operatorname{Re}(2) 96.702$ \\
\hline $\mathrm{TC}(1)-\mathrm{Cl}(4)-\mathrm{TC}(2) 97.582$ & $\operatorname{Re}(1)-\operatorname{Br}(4)-\operatorname{Re}(2) 96.566$ \\
\hline $\mathrm{TC}(2)-\mathrm{N}(6)-\mathrm{O}(7) \quad 176.336$ & $\operatorname{Re}(2)-\mathrm{N}(6)-\mathrm{O}(7) \quad 177.047$ \\
\hline $\mathrm{TC}(1)-\mathrm{N}(8)-\mathrm{O}(15)$ & $\operatorname{Re}(1)-\mathrm{N}(8)-\mathrm{O}(15)$ \\
\hline $\mathrm{TC}(2)-\mathrm{C}(11)-\mathrm{O}(9) \quad 178.450$ & $\operatorname{Re}(2)-C(11)-O(9) \quad 178.584$ \\
\hline $\mathrm{TC}(1)-\mathrm{C}(12)-\mathrm{O}(10) 179.368$ & $\operatorname{Re}(1)-C(12)-O(10) 178.266$ \\
\hline $\mathrm{TC}(2)-\mathrm{C}(16)-\mathrm{O}(13) 179.368$ & $\operatorname{Re}(2)-C(16)-O(13) 179.292$ \\
\hline $\mathrm{TC}(1)-\mathrm{C}(18)-\mathrm{O}(17) 178.950$ & $\operatorname{Re}(1)-C(18)-0(17) 180.000$ \\
\hline
\end{tabular}

\section{Isomer 2:}

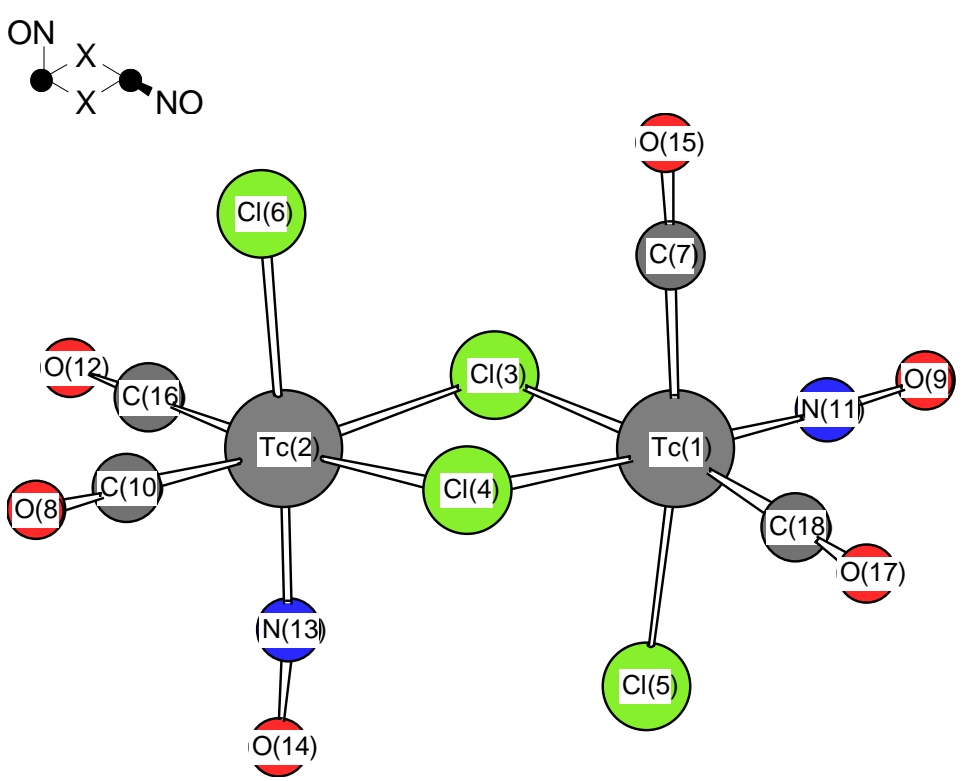

Bond lengths in $\AA$

\begin{tabular}{|c|c|}
\hline $\mathrm{M}=\mathrm{TC} ; \quad \mathrm{X}=\mathrm{Cl}$ & $\mathrm{M}=\mathrm{Re} \quad ; \mathrm{X}=\mathrm{Br}$ \\
\hline $\mathrm{TC}(1)-\mathrm{Cl}(3) 2.531$ & $\operatorname{Re}(1)-\operatorname{Br}(3) 2.699$ \\
\hline $\mathrm{TC}(1)-\mathrm{Cl}(4) 2.501$ & $\operatorname{Re}(1)-\operatorname{Br}(4) 2.672$ \\
\hline $\mathrm{TC}(1)-\mathrm{Cl}(5) 2.423$ & $\operatorname{Re}(1)-\operatorname{Br}(5) 2.592$ \\
\hline $\mathrm{TC}(1)-\mathrm{C}(7) \quad 1.994$ & $\operatorname{Re}(1)-C(7) \quad 1.977$ \\
\hline $\mathrm{TC}(1)-\mathrm{N}(11) \quad 1.799$ & $\operatorname{Re}(1)-N(11) \quad 1.797$ \\
\hline $\mathrm{TC}(1)-\mathrm{C}(18) 1.962$ & $\operatorname{Re}(1)-C(18) 1.949$ \\
\hline $\mathrm{TC}(2)-\mathrm{Cl}(3) \quad 2.524$ & $\operatorname{Re}(2)-\operatorname{Br}(3) 2.689$ \\
\hline $\mathrm{TC}(2)-\mathrm{Cl}(4) 2.549$ & $\operatorname{Re}(2)-\operatorname{Br}(4) 2.711$ \\
\hline $\operatorname{TC}(2)-\mathrm{Cl}(6) 2.386$ & $\operatorname{Re}(2)-\operatorname{Br}(6) 2.561$ \\
\hline $\mathrm{TC}(2)-\mathrm{C}(10) 1.961$ & $\operatorname{Re}(2)-C(10) 1.950$ \\
\hline $\mathrm{TC}(2)-\mathrm{N}(13) \quad 1.820$ & $\operatorname{Re}(2)-N(13) 1.816$ \\
\hline
\end{tabular}




\begin{tabular}{|c|c|c|}
\hline $\mathrm{TC}(2)-\mathrm{C}(16)$ & 1.956 & $\operatorname{Re}(2)-C(16) 1.945$ \\
\hline$C(7)-O(15)$ & 1.151 & $C(7)-O(15) \quad 1.154$ \\
\hline $\mathrm{O}(8)-\mathrm{C}(10)$ & 1.153 & $\mathrm{O}(8)-\mathrm{C}(10)$ \\
\hline $\mathrm{O}(9)-\mathrm{N}(11)$ & 1.167 & $\mathrm{O}(9)-\mathrm{N}(11)$ \\
\hline $\mathrm{O}(12)-\mathrm{C}(16)$ & 1.150 & $O(12)-C(16) 1.153$ \\
\hline $\mathrm{N}(13)-\mathrm{O}(14)$ & 1.166 & $\mathrm{~N}(13)-\mathrm{O}(14) 1.170$ \\
\hline $\mathrm{O}(17)-\mathrm{C}(18)$ & 1.151 & $\mathrm{O}(17)-\mathrm{C}(18) \quad 1.154$ \\
\hline
\end{tabular}

Bond angles in degrees

\begin{tabular}{|c|c|c|}
\hline \multicolumn{2}{|l|}{$\mathrm{M}=\mathrm{TC} ; \mathrm{X}=\mathrm{Cl}$} & $\mathrm{M}=\mathrm{Re} ; \mathrm{X}=\mathrm{Br}$ \\
\hline $\mathrm{Cl}(3)-\mathrm{TC}(1)-\mathrm{Cl}(4)$ & 84.169 & $\operatorname{Br}(3)-\operatorname{Re}(1)-\operatorname{Br}(4) 84.578$ \\
\hline $\mathrm{Cl}(3)-\mathrm{TC}(1)-\mathrm{Cl}(5)$ & 86.853 & $\operatorname{Br}(3)-\operatorname{Re}(1)-\operatorname{Br}(5) 87.106$ \\
\hline $\mathrm{Cl}(3)-\mathrm{TC}(1)-\mathrm{C}(7)$ & 88.528 & $\operatorname{Br}(3)-\operatorname{Re}(1)-C(7) \quad 88.934$ \\
\hline $\mathrm{Cl}(3)-\mathrm{TC}(1)-\mathrm{N}(11)$ & 95.867 & $\operatorname{Br}(3)-\operatorname{Re}(1)-N(11) 95.330$ \\
\hline $\mathrm{Cl}(3)-\mathrm{TC}(1)-\mathrm{C}(18)$ & 171.564 & $\operatorname{Br}(3)-\operatorname{Re}(1)-C(18) 172.293$ \\
\hline $\mathrm{Cl}(4)-\mathrm{TC}(1)-\mathrm{Cl}(5)$ & 87.928 & $\operatorname{Br}(4)-\operatorname{Re}(1)-\operatorname{Br}(5) 88.876$ \\
\hline $\mathrm{Cl}(4)-\mathrm{TC}(1)-\mathrm{C}(7)$ & 84.150 & $\operatorname{Br}(4)-\operatorname{Re}(1)-C(7) \quad 83.984$ \\
\hline $\mathrm{Cl}(4)-\mathrm{TC}(1)-\mathrm{N}(11)$ & 176.883 & $\operatorname{Br}(4)-\operatorname{Re}(1)-\mathrm{N}(11) 176.136$ \\
\hline $\mathrm{Cl}(4)-\mathrm{TC}(1)-\mathrm{C}(18)$ & 88.673 & $\operatorname{Br}(4)-\operatorname{Re}(1)-C(18) 88.775$ \\
\hline $\mathrm{Cl}(5)-\mathrm{TC}(1)-\mathrm{C}(7)$ & 171.197 & $\operatorname{Br}(5)-\operatorname{Re}(1)-C(7) \quad 172.137$ \\
\hline $\mathrm{Cl}(5)-\mathrm{TC}(1)-\mathrm{N}(11)$ & 95.185 & $\operatorname{Br}(5)-\operatorname{Re}(1)-\mathrm{N}(11) 94.985$ \\
\hline $\mathrm{Cl}(5)-\mathrm{TC}(1)-\mathrm{C}(18)$ & 88.441 & $\operatorname{Br}(5)-\operatorname{Re}(1)-C(18) 88.871$ \\
\hline $\mathrm{C}(7)-\mathrm{TC}(1)-\mathrm{N}(11)$ & 92.735 & $C(7)-\operatorname{Re}(1)-N(11) \quad 92.146$ \\
\hline $\mathrm{C}(7)-\mathrm{TC}(1)-\mathrm{C}(18)$ & 95.190 & $C(7)-\operatorname{Re}(1)-C(18) \quad 94.254$ \\
\hline $\mathrm{N}(11)-\mathrm{TC}(1)-\mathrm{C}(18)$ & 91.521 & $\mathrm{~N}(11)-\operatorname{Re}(1)-\mathrm{C}(18) 91.565$ \\
\hline $\mathrm{Cl}(3)-\mathrm{TC}(2)-\mathrm{Cl}(4)$ & 83.328 & $\operatorname{Br}(3)-\operatorname{Re}(2)-\operatorname{Br}(4) 84.015$ \\
\hline $\mathrm{Cl}(3)-\mathrm{TC}(2)-\mathrm{Cl}(6)$ & 89.074 & $\operatorname{Br}(3)-\operatorname{Re}(2)-\operatorname{Br}(6) 89.554$ \\
\hline $\mathrm{Cl}(3)-\mathrm{TC}(2)-\mathrm{C}(10)$ & 172.338 & $\operatorname{Br}(3)-\operatorname{Re}(2)-C(10) 173.417$ \\
\hline $\mathrm{Cl}(3)-\mathrm{TC}(2)-\mathrm{N}(13)$ & 94.029 & $\operatorname{Br}(3)-\operatorname{Re}(2)-\mathrm{N}(13) 93.371$ \\
\hline $\mathrm{Cl}(3)-\mathrm{TC}(2)-\mathrm{C}(16)$ & 92.987 & $\operatorname{Br}(3)-\operatorname{Re}(2)-C(16) 92.483$ \\
\hline $\mathrm{Cl}(4)-\mathrm{TC}(2)-\mathrm{Cl}(6)$ & 88.056 & $\operatorname{Br}(4)-\operatorname{Re}(2)-\operatorname{Br}(6) 88.607$ \\
\hline $\mathrm{Cl}(4)-\mathrm{TC}(2)-\mathrm{C}(10)$ & 92.340 & $\operatorname{Br}(4)-\operatorname{Re}(2)-C(10) 92.193$ \\
\hline $\mathrm{Cl}(4)-\mathrm{TC}(2)-\mathrm{N}(13)$ & 93.490 & $\operatorname{Br}(4)-\operatorname{Re}(2)-\mathrm{N}(13) 92.849$ \\
\hline $\mathrm{Cl}(4)-\mathrm{TC}(2)-\mathrm{C}(16)$ & 173.739 & $\operatorname{Br}(4)-\operatorname{Re}(2)-C(16) 174.153$ \\
\hline $\mathrm{Cl}(6)-\mathrm{TC}(2)-\mathrm{C}(10)$ & 84.452 & $\operatorname{Br}(6)-\operatorname{Re}(2)-C(10) 84.970$ \\
\hline $\mathrm{Cl}(6)-\mathrm{TC}(2)-\mathrm{N}(13)$ & 176.664 & $\operatorname{Br}(6)-\operatorname{Re}(2)-\mathrm{N}(13) 176.867$ \\
\hline $\mathrm{Cl}(6)-\mathrm{TC}(2)-\mathrm{C}(16)$ & 86.825 & $\operatorname{Br}(6)-\operatorname{Re}(2)-C(16) 86.680$ \\
\hline $\mathrm{C}(10)-\mathrm{TC}(2)-\mathrm{N}(13)$ & 92.536 & $C(10)-\operatorname{Re}(2)-N(13) 92.182$ \\
\hline$C(10)-T C(2)-C(16)$ & 90.747 & $C(10)-\operatorname{Re}(2)-C(16) 90.838$ \\
\hline$N(13)-T C(2)-C(16)$ & 91.808 & $N(13)-\operatorname{Re}(2)-C(16) 92.029$ \\
\hline $\mathrm{TC}(1)-\mathrm{Cl}(3)-\mathrm{TC}(2)$ & 95.699 & $\operatorname{Re}(1)-\operatorname{Br}(3)-\operatorname{Re}(2) 95.183$ \\
\hline $\mathrm{TC}(1)-\mathrm{Cl}(4)-\mathrm{TC}(2)$ & 95.821 & $\operatorname{Re}(1)-\operatorname{Br}(4)-\operatorname{Re}(2) 95.270$ \\
\hline $\operatorname{TC}(1)-C(7)-O(15)$ & 179.292 & $\operatorname{Re}(1)-C(7)-O(15) \quad 177.696$ \\
\hline $\mathrm{TC}(2)-\mathrm{C}(10)-\mathrm{O}(8)$ & 180.000 & $\operatorname{Re}(2)-C(10)-O(8)$ \\
\hline $\operatorname{TC}(1)-N(11)-O(9)$ & 178.075 & $\operatorname{Re}(1)-\mathrm{N}(11)-\mathrm{O}(9) \quad 178.154$ \\
\hline $\mathrm{TC}(2)-\mathrm{N}(13)-\mathrm{O}(14)$ & 174.059 & $\operatorname{Re}(2)-\mathrm{N}(13)-\mathrm{O}(14) 175.189$ \\
\hline $\mathrm{TC}(2)-\mathrm{C}(16)-\mathrm{O}(12)$ & 178.735 & $\operatorname{Re}(2)-C(16)-O(12) 178.694$ \\
\hline $\mathrm{TC}(1)-\mathrm{C}(18)-\mathrm{O}(17)$ & 178.999 & $\operatorname{Re}(1)-C(18)-0(17) 180.000$ \\
\hline
\end{tabular}




\section{Isomer 3:}<smiles>[X]C(O)[14C]([X])([X])O[Na]</smiles>

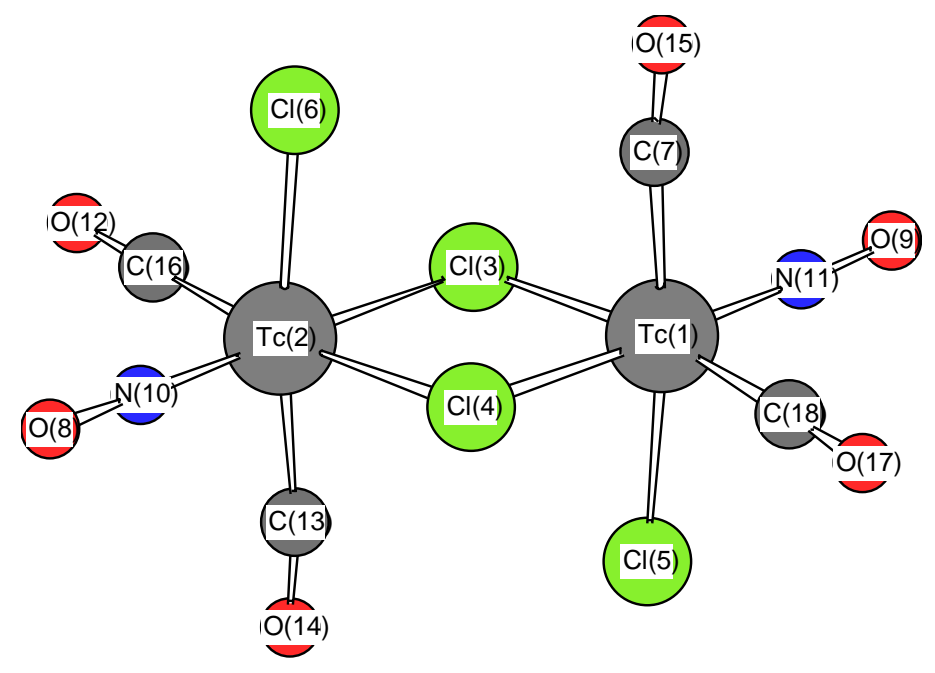

Bond lengths in $\AA$

\begin{tabular}{|c|c|}
\hline $\mathrm{M}=\mathrm{TC} ; \quad \mathrm{X}=\mathrm{Cl}$ & $\mathrm{M}=\mathrm{Re} \quad ; \mathrm{X}=\mathrm{Br}$ \\
\hline $\mathrm{TC}(1)-\mathrm{Cl}(3) 2.546$ & $\operatorname{Re}(1)-\operatorname{Br}(3) 2.711$ \\
\hline $\mathrm{TC}(1)-\mathrm{Cl}(4) 2.492$ & $\operatorname{Re}(1)-\operatorname{Br}(4) 2.664$ \\
\hline $\operatorname{TC}(1)-\mathrm{Cl}(5) 2.420$ & $\operatorname{Re}(1)-\operatorname{Br}(5) 2.593$ \\
\hline $\mathrm{TC}(1)-\mathrm{C}(7) \quad 1.999$ & $\operatorname{Re}(1)-C(7) \quad 1.979$ \\
\hline $\mathrm{TC}(1)-\mathrm{N}(11) 1.796$ & $\operatorname{Re}(1)-N(11) 1.796$ \\
\hline $\mathrm{TC}(1)-\mathrm{C}(18) 1.958$ & $\operatorname{Re}(1)-C(18) \quad 1.947$ \\
\hline $\mathrm{TC}(2)-\mathrm{Cl}(3) 2.502$ & $\operatorname{Re}(2)-\operatorname{Br}(3) 2.672$ \\
\hline $\operatorname{TC}(2)-\mathrm{Cl}(4) 2.553$ & $\operatorname{Re}(2)-\operatorname{Br}(4) 2.720$ \\
\hline $\operatorname{TC}(2)-\mathrm{Cl}(6) 2.419$ & $\operatorname{Re}(2)-\operatorname{Br}(6) 2.589$ \\
\hline $\mathrm{TC}(2)-\mathrm{N}(10) \quad 1.797$ & $\operatorname{Re}(2)-N(10) 1.795$ \\
\hline $\mathrm{TC}(2)-\mathrm{C}(13) 1.998$ & $\operatorname{Re}(2)-C(13) 1.982$ \\
\hline $\mathrm{TC}(2)-\mathrm{C}(16) \quad 1.954$ & $\operatorname{Re}(2)-C(16) 1.943$ \\
\hline$C(7)-O(15) \quad 1.150$ & $C(7)-O(15) \quad 1.152$ \\
\hline $\mathrm{O}(8)-\mathrm{N}(10)$ & $\mathrm{O}(8)-\mathrm{N}(10)$ \\
\hline $\mathrm{O}(9)-\mathrm{N}(11) \quad 1.165$ & $\mathrm{O}(9)-\mathrm{N}(11) \quad 1.172$ \\
\hline $\mathrm{O}(12)-\mathrm{C}(16) 1.151$ & $\mathrm{O}(12)-\mathrm{C}(16) 1.154$ \\
\hline$C(13)-O(14) 1.149$ & $C(13)-O(14) 1.152$ \\
\hline $\mathrm{O}(17)-\mathrm{C}(18) 1.151$ & $\mathrm{O}(17)-\mathrm{C}(18) 1.154$ \\
\hline
\end{tabular}

Bond angles in degrees

\begin{tabular}{|l|l|}
\hline $\mathrm{M}=\mathrm{TC} ; \mathrm{X}=\mathrm{Cl}$ & $\mathrm{M}=\mathrm{Re} ; \mathrm{X}=\mathrm{Br}$ \\
\hline $\mathrm{Cl}(3)-\mathrm{TC}(1)-\mathrm{Cl}(4) 85.208$ & $\mathrm{Br}(3)-\operatorname{Re}(1)-\mathrm{Br}(4) 85.391$ \\
$\mathrm{Cl}(3)-\mathrm{TC}(1)-\mathrm{Cl}(5) 85.590$ & $\mathrm{Br}(3)-\mathrm{Re}(1)-\mathrm{Br}(5) 86.250$ \\
$\mathrm{Cl}(3)-\mathrm{TC}(1)-\mathrm{C}(7) 91.287$ & $\mathrm{Br}(3)-\operatorname{Re}(1)-\mathrm{C}(7) 90.652$ \\
$\mathrm{Cl}(3)-\mathrm{TC}(1)-\mathrm{N}(11) 95.078$ & $\mathrm{Br}(3)-\operatorname{Re}(1)-\mathrm{N}(11) 94.681$ \\
$\mathrm{Cl}(3)-\mathrm{TC}(1)-\mathrm{C}(18) 171.100$ & $\mathrm{Br}(3)-\operatorname{Re}(1)-\mathrm{C}(18) 172.069$ \\
$\mathrm{Cl}(4)-\mathrm{TC}(1)-\mathrm{Cl}(5) 87.673$ & $\mathrm{Br}(4)-\operatorname{Re}(1)-\mathrm{Br}(5) 88.930$ \\
$\mathrm{Cl}(4)-\mathrm{TC}(1)-\mathrm{C}(7) 87.052$ & $\mathrm{Br}(4)-\operatorname{Re}(1)-\mathrm{C}(7) 85.792$ \\
$\mathrm{Cl}(4)-\mathrm{TC}(1)-\mathrm{N}(11) 177.717$ & $\mathrm{Br}(4)-\operatorname{Re}(1)-\mathrm{N}(11) 176.818$ \\
$\mathrm{Cl}(4)-\mathrm{TC}(1)-\mathrm{C}(18) 88.079$ & $\mathrm{Br}(4)-\operatorname{Re}(1)-\mathrm{C}(18) 88.304$ \\
$\mathrm{Cl}(5)-\mathrm{TC}(1)-\mathrm{C}(7) 174.076$ & $\mathrm{Br}(5)-\operatorname{Re}(1)-\mathrm{C}(7) 174.085$ \\
$\mathrm{Cl}(5)-\mathrm{TC}(1)-\mathrm{N}(11) 94.602$ & $\mathrm{Br}(5)-\operatorname{Re}(1)-\mathrm{N}(11) 94.240$ \\
\hline
\end{tabular}




\begin{tabular}{|c|c|c|c|}
\hline $\mathrm{Cl}(5)-\mathrm{TC}(1)-\mathrm{C}(18)$ & 88.313 & $\operatorname{Br}(5)-\operatorname{Re}(1)-C(18)$ & 88.834 \\
\hline $\mathrm{C}(7)-\mathrm{TC}(1)-\mathrm{N}(11)$ & 90.680 & $C(7)-\operatorname{Re}(1)-N(11)$ & 91.035 \\
\hline $\mathrm{C}(7)-\mathrm{TC}(1)-\mathrm{C}(18)$ & 94.198 & $C(7)-\operatorname{Re}(1)-C(18)$ & 93.677 \\
\hline $\mathrm{N}(11)-\mathrm{TC}(1)-\mathrm{C}(18)$ & 91.861 & $N(11)-\operatorname{Re}(1)-C(18)$ & 91.880 \\
\hline $\mathrm{Cl}(3)-\mathrm{TC}(2)-\mathrm{Cl}(4)$ & 84.854 & $\operatorname{Br}(3)-\operatorname{Re}(2)-\operatorname{Br}(4)$ & 85.066 \\
\hline $\mathrm{Cl}(3)-\mathrm{TC}(2)-\mathrm{Cl}(6)$ & 86.904 & $\operatorname{Br}(3)-\operatorname{Re}(2)-\operatorname{Br}(6)$ & 88.278 \\
\hline $\mathrm{Cl}(3)-\mathrm{TC}(2)-\mathrm{N}(10)$ & 178.182 & $\operatorname{Br}(3)-\operatorname{Re}(2)-\mathrm{N}(10)$ & 177.488 \\
\hline $\mathrm{Cl}(3)-\mathrm{TC}(2)-\mathrm{C}(13)$ & 85.823 & $\operatorname{Br}(3)-\operatorname{Re}(2)-C(13)$ & 85.265 \\
\hline $\mathrm{Cl}(3)-\mathrm{TC}(2)-\mathrm{C}(16)$ & 88.566 & $\operatorname{Br}(3)-\operatorname{Re}(2)-C(16)$ & 88.780 \\
\hline $\mathrm{Cl}(4)-\mathrm{TC}(2)-\mathrm{Cl}(6)$ & 86.540 & $\operatorname{Br}(4)-\operatorname{Re}(2)-\operatorname{Br}(6)$ & 86.867 \\
\hline $\mathrm{Cl}(4)-\mathrm{TC}(2)-\mathrm{N}(10)$ & 95.274 & $\operatorname{Br}(4)-\operatorname{Re}(2)-N(10)$ & 94.907 \\
\hline $\mathrm{Cl}(4)-\mathrm{TC}(2)-\mathrm{C}(13)$ & 90.623 & $\operatorname{Br}(4)-\operatorname{Re}(2)-C(13)$ & 90.588 \\
\hline $\mathrm{Cl}(4)-\mathrm{TC}(2)-\mathrm{C}(16)$ & 172.248 & $\operatorname{Br}(4)-\operatorname{Re}(2)-C(16)$ & 172.702 \\
\hline $\mathrm{Cl}(6)-\mathrm{TC}(2)-\mathrm{N}(10)$ & 94.924 & $\operatorname{Br}(6)-\operatorname{Re}(2)-N(10)$ & 94.230 \\
\hline $\mathrm{Cl}(6)-\mathrm{TC}(2)-\mathrm{C}(13)$ & 172.405 & $\operatorname{Br}(6)-\operatorname{Re}(2)-C(13)$ & 173.244 \\
\hline $\mathrm{Cl}(6)-\mathrm{TC}(2)-\mathrm{C}(16)$ & 89.014 & $\operatorname{Br}(6)-\operatorname{Re}(2)-C(16)$ & 89.032 \\
\hline$N(10)-T C(2)-C(13)$ & 92.352 & $N(10)-\operatorname{Re}(2)-C(13)$ & 92.221 \\
\hline $\mathrm{N}(10)-\mathrm{TC}(2)-\mathrm{C}(16)$ & 91.432 & $N(10)-\operatorname{Re}(2)-C(16)$ & 91.413 \\
\hline$C(13)-T C(2)-C(16)$ & 92.992 & $C(13)-\operatorname{Re}(2)-C(16)$ & 92.815 \\
\hline $\mathrm{TC}(1)-\mathrm{Cl}(3)-\mathrm{TC}(2)$ & 94.942 & $\operatorname{Re}(1)-\operatorname{Br}(3)-\operatorname{Re}(2)$ & 94.788 \\
\hline $\mathrm{TC}(1)-\mathrm{Cl}(4)-\mathrm{TC}(2)$ & 94.998 & $\operatorname{Re}(1)-\operatorname{Br}(4)-\operatorname{Re}(2)$ & 94.755 \\
\hline $\mathrm{TC}(1)-\mathrm{C}(7)-\mathrm{O}(15)$ & 174.507 & $\operatorname{Re}(1)-C(7)-O(15)$ & 174.855 \\
\hline $\mathrm{TC}(2)-\mathrm{N}(10)-\mathrm{O}(8)$ & 178.859 & $\operatorname{Re}(2)-N(10)-O(8)$ & 178.355 \\
\hline $\mathrm{TC}(1)-\mathrm{N}(11)-\mathrm{O}(9)$ & 178.450 & $\operatorname{Re}(1)-N(11)-O(9)$ & 178.902 \\
\hline $\mathrm{TC}(2)-\mathrm{C}(13)-\mathrm{O}(14)$ & 174.426 & $\operatorname{Re}(2)-C(13)-O(14)$ & 175.003 \\
\hline $\mathrm{TC}(2)-\mathrm{C}(16)-\mathrm{O}(12)$ & 178.735 & $\operatorname{Re}(2)-C(16)-O(12)$ & 179.553 \\
\hline $\mathrm{TC}(1)-\mathrm{C}(18)-\mathrm{O}(17)$ & 178.418 & $\operatorname{Re}(1)-C(18)-O(17)$ & 179.684 \\
\hline
\end{tabular}




\section{Isomer 4:}

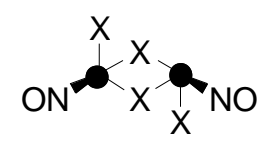

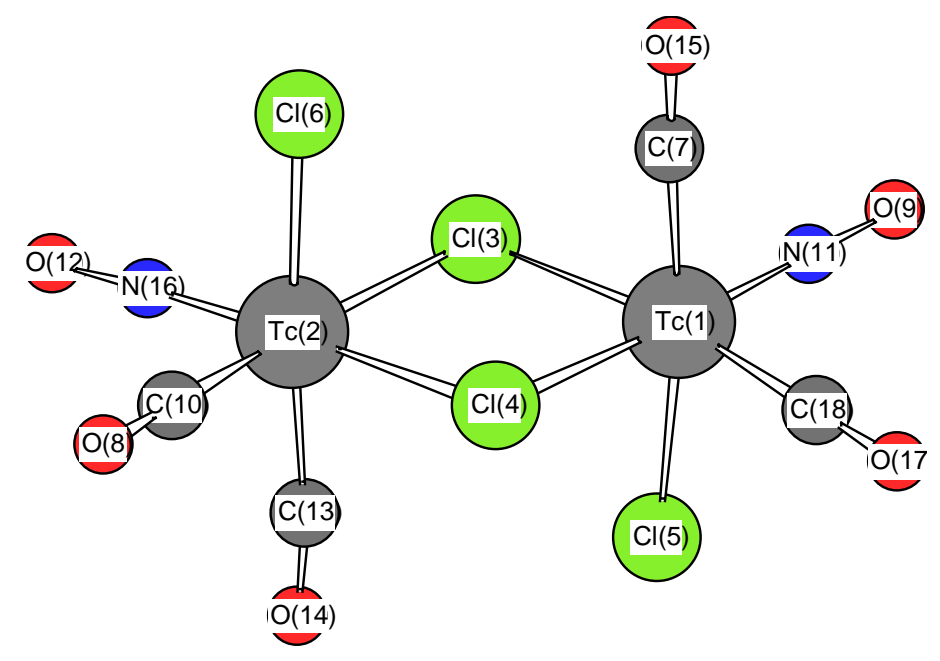

Bond lengths in $\AA$

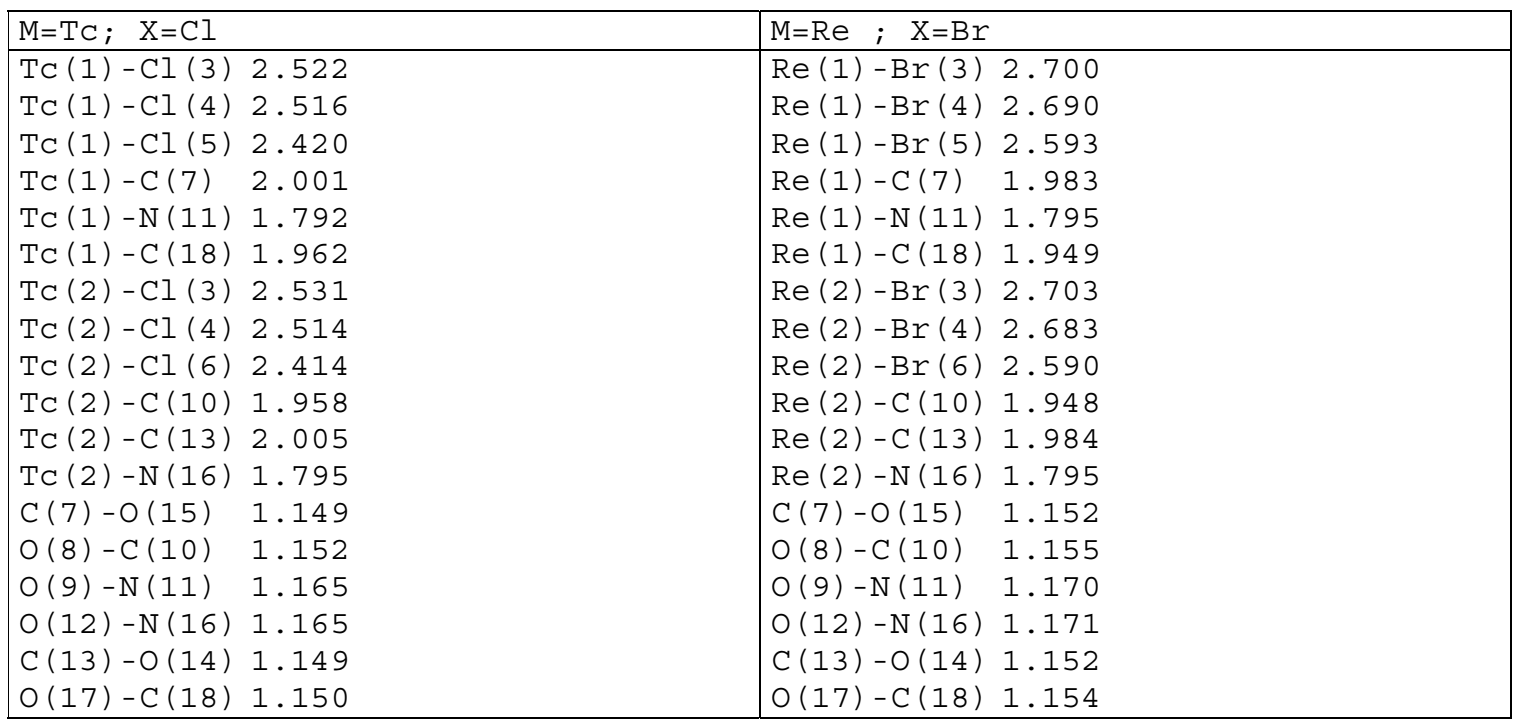

Bond angles in degrees

\begin{tabular}{|l|l|}
\hline $\mathrm{M}=\mathrm{TC} ; \mathrm{X}=\mathrm{Cl}$ & $\mathrm{M}=\operatorname{Re} ; \mathrm{X}=\mathrm{Br}$ \\
\hline $\mathrm{Cl}(3)-\mathrm{TC}(1)-\mathrm{Cl}(4) 83.700$ & $\mathrm{Br}(3)-\operatorname{Re}(1)-\mathrm{Br}(4) 84.400$ \\
$\mathrm{Cl}(3)-\mathrm{TC}(1)-\mathrm{Cl}(5) 87.306$ & $\mathrm{Br}(3)-\operatorname{Re}(1)-\mathrm{Br}(5) 87.299$ \\
$\mathrm{Cl}(3)-\mathrm{TC}(1)-\mathrm{C}(7) 89.154$ & $\mathrm{Br}(3)-\operatorname{Re}(1)-\mathrm{C}(7) 89.512$ \\
$\mathrm{Cl}(3)-\mathrm{TC}(1)-\mathrm{N}(11) 96.024$ & $\operatorname{Br}(3)-\operatorname{Re}(1)-\mathrm{N}(11) 95.090$ \\
$\mathrm{Cl}(3)-\mathrm{TC}(1)-\mathrm{C}(18) 171.045$ & $\operatorname{Br}(3)-\operatorname{Re}(1)-\mathrm{C}(18) 172.241$ \\
$\mathrm{Cl}(4)-\mathrm{TC}(1)-\mathrm{Cl}(5) 87.481$ & $\operatorname{Br}(4)-\operatorname{Re}(1)-\mathrm{Br}(5) 88.507$ \\
$\mathrm{Cl}(4)-\mathrm{TC}(1)-\mathrm{C}(7) 85.349$ & $\operatorname{Br}(4)-\operatorname{Re}(1)-\mathrm{C}(7) 84.704$ \\
$\mathrm{Cl}(4)-\mathrm{TC}(1)-\mathrm{N}(11) 177.610$ & $\operatorname{Br}(4)-\operatorname{Re}(1)-\mathrm{N}(11) 176.818$ \\
$\mathrm{Cl}(4)-\mathrm{TC}(1)-\mathrm{C}(18) 88.533$ & $\operatorname{Br}(4)-\operatorname{Re}(1)-\mathrm{C}(18) 88.876$ \\
$\mathrm{Cl}(5)-\mathrm{TC}(1)-\mathrm{C}(7) 172.312$ & $\operatorname{Br}(5)-\operatorname{Re}(1)-\mathrm{C}(7) 172.756$ \\
\hline
\end{tabular}




\begin{tabular}{|c|c|c|}
\hline $\mathrm{Cl}(5)-\mathrm{TC}(1)-\mathrm{N}(11)$ & 94.886 & $\operatorname{Br}(5)-\operatorname{Re}(1)-\mathrm{N}(11) 94.606$ \\
\hline $\mathrm{Cl}(5)-\mathrm{TC}(1)-\mathrm{C}(18)$ & 87.907 & $\operatorname{Br}(5)-\operatorname{Re}(1)-C(18) 88.661$ \\
\hline $\mathrm{C}(7)-\mathrm{TC}(1)-\mathrm{N}(11)$ & 92.270 & $C(7)-\operatorname{Re}(1)-N(11) \quad 92.156$ \\
\hline $\mathrm{C}(7)-\mathrm{TC}(1)-\mathrm{C}(18)$ & 94.669 & $C(7)-\operatorname{Re}(1)-C(18)$ \\
\hline $\mathrm{N}(11)-\mathrm{TC}(1)-\mathrm{C}(18)$ & 91.918 & $\mathrm{~N}(11)-\operatorname{Re}(1)-\mathrm{C}(18) 91.833$ \\
\hline $\mathrm{Cl}(3)-\mathrm{TC}(2)-\mathrm{Cl}(4)$ & 83.571 & $\operatorname{Br}(3)-\operatorname{Re}(2)-\operatorname{Br}(4) 84.464$ \\
\hline $\mathrm{Cl}(3)-\mathrm{TC}(2)-\mathrm{Cl}(6)$ & 87.085 & $\operatorname{Br}(3)-\operatorname{Re}(2)-\operatorname{Br}(6) 86.725$ \\
\hline $\mathrm{Cl}(3)-\mathrm{TC}(2)-\mathrm{C}(10)$ & 170.989 & $\operatorname{Br}(3)-\operatorname{Re}(2)-C(10) 171.975$ \\
\hline $\mathrm{Cl}(3)-\mathrm{TC}(2)-\mathrm{C}(13)$ & 89.035 & $\operatorname{Br}(3)-\operatorname{Re}(2)-C(13) 89.109$ \\
\hline $\mathrm{Cl}(3)-\mathrm{TC}(2)-\mathrm{N}(16)$ & 96.842 & $\operatorname{Br}(3)-\operatorname{Re}(2)-N(16) 95.942$ \\
\hline $\mathrm{Cl}(4)-\mathrm{TC}(2)-\mathrm{Cl}(6)$ & 88.531 & $\operatorname{Br}(4)-\operatorname{Re}(2)-\operatorname{Br}(6) 89.049$ \\
\hline $\mathrm{Cl}(4)-\mathrm{TC}(2)-\mathrm{C}(10)$ & 87.995 & $\operatorname{Br}(4)-\operatorname{Re}(2)-C(10) 88.045$ \\
\hline $\mathrm{Cl}(4)-\mathrm{TC}(2)-\mathrm{C}(13)$ & 87.970 & $\operatorname{Br}(4)-\operatorname{Re}(2)-C(13) 86.765$ \\
\hline $\mathrm{Cl}(4)-\mathrm{TC}(2)-\mathrm{N}(16)$ & 177.315 & $\operatorname{Br}(4)-\operatorname{Re}(2)-N(16) 176.635$ \\
\hline $\mathrm{Cl}(6)-\mathrm{TC}(2)-\mathrm{C}(10)$ & 89.540 & $\operatorname{Br}(6)-\operatorname{Re}(2)-C(10) 90.271$ \\
\hline $\mathrm{Cl}(6)-\mathrm{TC}(2)-\mathrm{C}(13)$ & 175.043 & $\operatorname{Br}(6)-\operatorname{Re}(2)-C(13) 174.361$ \\
\hline $\mathrm{Cl}(6)-\mathrm{TC}(2)-\mathrm{N}(16)$ & 94.146 & $\operatorname{Br}(6)-\operatorname{Re}(2)-N(16) 94.300$ \\
\hline$C(10)-T C(2)-C(13)$ & 93.844 & $C(10)-\operatorname{Re}(2)-C(13) 93.359$ \\
\hline $\mathrm{C}(10)-\mathrm{TC}(2)-\mathrm{N}(16)$ & 91.733 & $C(10)-\operatorname{Re}(2)-N(16) 91.703$ \\
\hline $\mathrm{C}(13)-\mathrm{TC}(2)-\mathrm{N}(16)$ & 89.374 & $C(13)-\operatorname{Re}(2)-N(16) 89.908$ \\
\hline $\mathrm{TC}(1)-\mathrm{Cl}(3)-\mathrm{TC}(2)$ & 96.036 & $\operatorname{Re}(1)-\operatorname{Br}(3)-\operatorname{Re}(2) 95.171$ \\
\hline $\mathrm{TC}(1)-\mathrm{Cl}(4)-\mathrm{TC}(2)$ & 96.615 & $\operatorname{Re}(1)-\operatorname{Br}(4)-\operatorname{Re}(2) 95.865$ \\
\hline $\mathrm{TC}(1)-\mathrm{C}(7)-\mathrm{O}(15)$ & 176.650 & $\operatorname{Re}(1)-C(7)-O(15) \quad 176.350$ \\
\hline $\mathrm{TC}(2)-\mathrm{C}(10)-\mathrm{O}(8)$ & 178.950 & $\operatorname{Re}(2)-C(10)-O(8)$ \\
\hline $\mathrm{TC}(1)-\mathrm{N}(11)-\mathrm{O}(9)$ & 178.902 & $\operatorname{Re}(1)-\mathrm{N}(11)-\mathrm{O}(9) \quad 178.549$ \\
\hline $\mathrm{TC}(2)-\mathrm{C}(13)-\mathrm{O}(14)$ & 172.833 & $\operatorname{Re}(2)-C(13)-O(14) 174.035$ \\
\hline $\mathrm{TC}(2)-\mathrm{N}(16)-\mathrm{O}(12)$ & 177.332 & $\operatorname{Re}(2)-N(16)-O(12) 178.387$ \\
\hline $\mathrm{TC}(1)-\mathrm{C}(18)-\mathrm{O}(17)$ & 178.859 & $\operatorname{Re}(1)-C(18)-O(17) 179.684$ \\
\hline
\end{tabular}




\section{Isomer 6:}

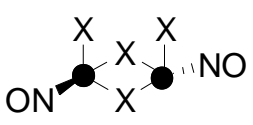

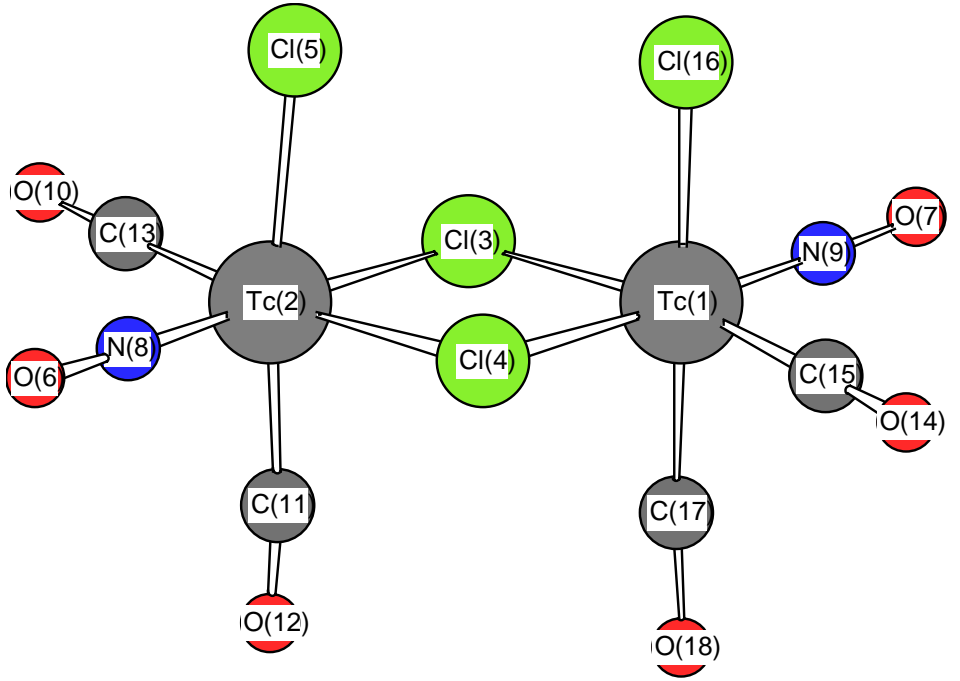

Bond lengths in $\AA$

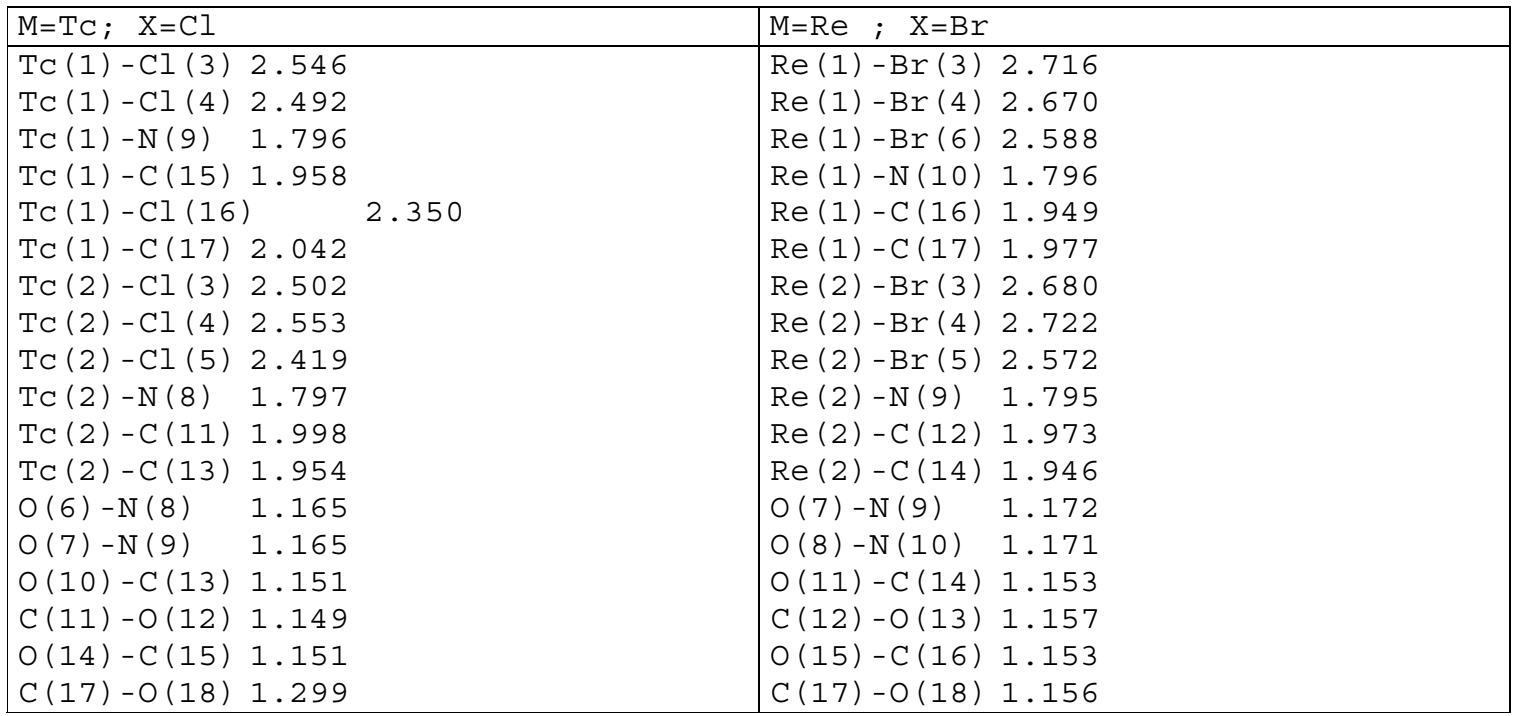

Bond angles in degrees

\begin{tabular}{|l|l|}
\hline $\mathrm{M}=\mathrm{TC} ; \mathrm{X}=\mathrm{Cl}$ & $\mathrm{M}=\mathrm{Re} ; \mathrm{X}=\mathrm{Br}$ \\
\hline $\mathrm{Cl}(3)-\mathrm{TC}(1)-\mathrm{Cl}(4) 85.208$ & $\mathrm{Br}(3)-\mathrm{Re}(1)-\mathrm{Br}(4) 83.770$ \\
$\mathrm{Cl}(3)-\mathrm{TC}(1)-\mathrm{N}(9) \quad 95.078$ & $\mathrm{Br}(3)-\mathrm{Re}(1)-\mathrm{Br}(6) 89.129$ \\
$\mathrm{Cl}(3)-\mathrm{TC}(1)-\mathrm{C}(15) 171.100$ & $\mathrm{Br}(3)-\operatorname{Re}(1)-\mathrm{N}(10) 94.753$ \\
$\mathrm{Cl}(3)-\mathrm{TC}(1)-\mathrm{Cl}(16) \quad 92.029$ & $\mathrm{Br}(3)-\operatorname{Re}(1)-\mathrm{C}(16) 171.949$ \\
$\mathrm{Cl}(3)-\mathrm{TC}(1)-\mathrm{C}(17) 88.259$ & $\mathrm{Br}(3)-\operatorname{Re}(1)-\mathrm{C}(17) 92.961$ \\
$\mathrm{Cl}(4)-\mathrm{TC}(1)-\mathrm{N}(9) \quad 177.717$ & $\mathrm{Br}(4)-\operatorname{Re}(1)-\mathrm{Br}(6) 89.505$ \\
$\mathrm{Cl}(4)-\mathrm{TC}(1)-\mathrm{C}(15) 88.079$ & $\mathrm{Br}(4)-\operatorname{Re}(1)-\mathrm{N}(10) 177.448$ \\
$\mathrm{Cl}(4)-\mathrm{TC}(1)-\mathrm{Cl}(16) \quad 88.722$ & $\mathrm{Br}(4)-\operatorname{Re}(1)-\mathrm{C}(16) 90.019$ \\
$\mathrm{Cl}(4)-\mathrm{TC}(1)-\mathrm{C}(17) 91.130$ & $\mathrm{Br}(4)-\operatorname{Re}(1)-\mathrm{C}(17) 86.021$ \\
$\mathrm{~N}(9)-\mathrm{TC}(1)-\mathrm{C}(15) 91.862$ & $\mathrm{Br}(6)-\operatorname{Re}(1)-\mathrm{N}(10) 92.551$ \\
$\mathrm{~N}(9)-\mathrm{TC}(1)-\mathrm{Cl}(16) 89.009$ & $\mathrm{Br}(6)-\operatorname{Re}(1)-\mathrm{C}(16) 85.688$ \\
\hline
\end{tabular}




\begin{tabular}{|c|c|}
\hline$N(9)-T C(1)-C(17)$ & $\operatorname{Br}(6)-\operatorname{Re}(1)-\mathrm{C}(17) 174.827$ \\
\hline $\mathrm{C}(15)-\mathrm{TC}(1)-\mathrm{Cl}(16) \quad 93.656$ & $N(10)-\operatorname{Re}(1)-C(16) 91.632$ \\
\hline$C(15)-T C(1)-C(17) 86.036$ & $N(10)-\operatorname{Re}(1)-C(17) 91.990$ \\
\hline $\mathrm{Cl}(16)-\mathrm{TC}(1)-\mathrm{C}(17) \quad 179.684$ & $C(16)-\operatorname{Re}(1)-C(17) 91.709$ \\
\hline $\mathrm{Cl}(3)-\mathrm{TC}(2)-\mathrm{Cl}(4) 84.856$ & $\operatorname{Br}(3)-\operatorname{Re}(2)-\operatorname{Br}(4) 83.476$ \\
\hline $\mathrm{Cl}(3)-\mathrm{TC}(2)-\mathrm{Cl}(5) 86.905$ & $\operatorname{Br}(3)-\operatorname{Re}(2)-\operatorname{Br}(5) 88.420$ \\
\hline $\mathrm{Cl}(3)-\mathrm{TC}(2)-\mathrm{N}(8) \quad 178.182$ & $\operatorname{Br}(3)-\operatorname{Re}(2)-N(9) \quad 176.883$ \\
\hline $\mathrm{Cl}(3)-\mathrm{TC}(2)-\mathrm{C}(11) 85.821$ & $\operatorname{Br}(3)-\operatorname{Re}(2)-C(12) 84.176$ \\
\hline $\mathrm{Cl}(3)-\mathrm{TC}(2)-\mathrm{C}(13) 88.565$ & $\operatorname{Br}(3)-\operatorname{Re}(2)-C(14) 88.806$ \\
\hline $\mathrm{Cl}(4)-\mathrm{TC}(2)-\mathrm{Cl}(5) 86.540$ & $\operatorname{Br}(4)-\operatorname{Re}(2)-\operatorname{Br}(5) 87.614$ \\
\hline $\mathrm{Cl}(4)-\mathrm{TC}(2)-\mathrm{N}(8) \quad 95.276$ & $\operatorname{Br}(4)-\operatorname{Re}(2)-\mathrm{N}(9) \quad 96.173$ \\
\hline $\mathrm{Cl}(4)-\mathrm{TC}(2)-\mathrm{C}(11) 90.623$ & $\operatorname{Br}(4)-\operatorname{Re}(2)-C(12) 90.210$ \\
\hline $\mathrm{Cl}(4)-\mathrm{TC}(2)-\mathrm{C}(13) 172.248$ & $\operatorname{Br}(4)-\operatorname{Re}(2)-C(14) 171.412$ \\
\hline $\mathrm{Cl}(5)-\mathrm{TC}(2)-\mathrm{N}(8) \quad 94.924$ & $\operatorname{Br}(5)-\operatorname{Re}(2)-\mathrm{N}(9) \quad 94.658$ \\
\hline $\mathrm{Cl}(5)-\mathrm{TC}(2)-\mathrm{C}(11) 172.398$ & $\operatorname{Br}(5)-\operatorname{Re}(2)-C(12) 172.471$ \\
\hline $\mathrm{Cl}(5)-\mathrm{TC}(2)-\mathrm{C}(13) 89.014$ & $\operatorname{Br}(5)-\operatorname{Re}(2)-C(14) 88.414$ \\
\hline $\mathrm{N}(8)-\mathrm{TC}(2)-\mathrm{C}(11) \quad 92.352$ & $N(9)-\operatorname{Re}(2)-C(12) \quad 92.738$ \\
\hline $\mathrm{N}(8)-\mathrm{TC}(2)-\mathrm{C}(13) \quad 91.431$ & $N(9)-\operatorname{Re}(2)-C(14) \quad 91.737$ \\
\hline $\mathrm{C}(11)-\mathrm{TC}(2)-\mathrm{C}(13) 92.992$ & $C(12)-\operatorname{Re}(2)-C(14) 92.758$ \\
\hline $\mathrm{TC}(1)-\mathrm{Cl}(3)-\mathrm{TC}(2) 94.940$ & $\operatorname{Re}(1)-\operatorname{Br}(3)-\operatorname{Re}(2) 96.069$ \\
\hline $\mathrm{TC}(1)-\mathrm{Cl}(4)-\mathrm{TC}(2) 94.998$ & $\operatorname{Re}(1)-\operatorname{Br}(4)-\operatorname{Re}(2) 96.136$ \\
\hline $\mathrm{TC}(2)-\mathrm{N}(8)-\mathrm{O}(6) \quad 178.859$ & $\operatorname{Re}(2)-N(9)-O(7) \quad 178.266$ \\
\hline $\mathrm{TC}(1)-\mathrm{N}(9)-\mathrm{O}(7) \quad 178.481$ & $\operatorname{Re}(1)-N(10)-O(8) \quad 178.815$ \\
\hline $\mathrm{TC}(2)-\mathrm{C}(11)-\mathrm{O}(12) 174.426$ & $\operatorname{Re}(2)-C(12)-O(13) 177.205$ \\
\hline $\mathrm{TC}(2)-\mathrm{C}(13)-\mathrm{O}(10) 178.735$ & $\operatorname{Re}(2)-C(14)-O(11) 180.000$ \\
\hline $\mathrm{TC}(1)-\mathrm{C}(15)-\mathrm{O}(14) 178.450$ & $\operatorname{Re}(1)-C(16)-O(15) 178.950$ \\
\hline $\mathrm{TC}(1)-\mathrm{C}(17)-\mathrm{O}(18) 176.446$ & $\operatorname{Re}(1)-C(17)-O(18) 175.545$ \\
\hline
\end{tabular}




\section{Isomer 7:}<smiles>[X]N(O)[X]([X])([X])[O]</smiles>

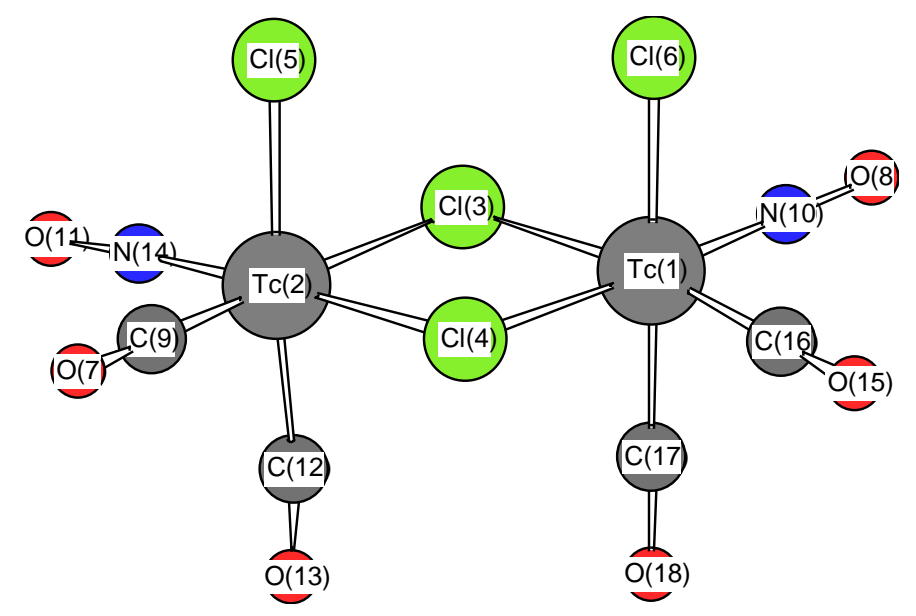

Bond lengths in $\AA$

\begin{tabular}{|c|c|c|}
\hline $\mathrm{M}=\mathrm{TC} ; \quad \mathrm{X}=\mathrm{Cl}$ & \multicolumn{2}{|c|}{$\mathrm{M}=\mathrm{Re} \quad ; \mathrm{X}=\mathrm{Br}$} \\
\hline $\mathrm{TC}(1)-\mathrm{Cl}(3) 2.522$ & $\operatorname{Re}(1)-\operatorname{Br}(3)$ & 2.708 \\
\hline $\mathrm{TC}(1)-\mathrm{Cl}(4) 2.516$ & $\operatorname{Re}(1)-\operatorname{Br}(4)$ & 2.703 \\
\hline $\mathrm{TC}(1)-\mathrm{Cl}(6) 2.350$ & $\operatorname{Re}(1)-\operatorname{Br}(6)$ & 2.573 \\
\hline $\mathrm{TC}(1)-\mathrm{N}(10) 1.792$ & $\operatorname{Re}(1)-\mathrm{N}(10)$ & 1.796 \\
\hline $\mathrm{TC}(1)-\mathrm{C}(16) 1.962$ & $\operatorname{Re}(1)-C(16)$ & 1.953 \\
\hline $\mathrm{TC}(1)-\mathrm{C}(17) 2.050$ & $\operatorname{Re}(1)-C(17)$ & 1.974 \\
\hline $\mathrm{TC}(2)-\mathrm{Cl}(3) 2.531$ & $\operatorname{Re}(2)-\operatorname{Br}(3)$ & 2.705 \\
\hline $\mathrm{TC}(2)-\mathrm{Cl}(4) 2.514$ & $\operatorname{Re}(2)-\operatorname{Br}(4)$ & 2.699 \\
\hline $\mathrm{TC}(2)-\mathrm{Cl}(5) 2.414$ & $\operatorname{Re}(2)-\operatorname{Br}(5)$ & 2.572 \\
\hline $\operatorname{TC}(2)-\mathrm{C}(9) \quad 1.958$ & $\operatorname{Re}(2)-C(9)$ & 1.950 \\
\hline $\operatorname{TC}(2)-\mathrm{C}(12) 2.005$ & $\operatorname{Re}(2)-C(12)$ & 1.974 \\
\hline $\mathrm{TC}(2)-\mathrm{N}(14) 1.795$ & $\operatorname{Re}(2)-\mathrm{N}(14)$ & 1.794 \\
\hline $\mathrm{O}(7)-\mathrm{C}(9) \quad 1.152$ & $\mathrm{O}(7)-\mathrm{C}(9)$ & 1.154 \\
\hline $\mathrm{O}(8)-\mathrm{N}(10) \quad 1.165$ & $O(8)-N(10)$ & 1.171 \\
\hline $\mathrm{O}(11)-\mathrm{N}(14) 1.165$ & $\mathrm{O}(11)-\mathrm{N}(14)$ & 1.172 \\
\hline$C(12)-O(13) 1.149$ & $C(12)-O(13)$ & 1.157 \\
\hline$O(15)-C(16) 1.150$ & $O(15)-C(16)$ & 1.152 \\
\hline$C(17)-O(18) 1.300$ & $C(17)-O(18)$ & 1.156 \\
\hline
\end{tabular}

Bond angles in degrees

\begin{tabular}{|l|l|}
\hline $\mathrm{M}=\mathrm{TC} ; \mathrm{X}=\mathrm{Cl}$ & $\mathrm{M}=\mathrm{Re} ; \mathrm{X}=\mathrm{Br}$ \\
\hline $\mathrm{Cl}(3)-\mathrm{TC}(1)-\mathrm{Cl}(4) 83.700$ & $\mathrm{Br}(3)-\operatorname{Re}(1)-\mathrm{Br}(4) 83.525$ \\
$\mathrm{C} 1(3)-\mathrm{TC}(1)-\mathrm{C} 1(6) 92.074$ & $\mathrm{Br}(3)-\operatorname{Re}(1)-\mathrm{Br}(6) 88.462$ \\
$\mathrm{C} 1(3)-\mathrm{TC}(1)-\mathrm{N}(10) 96.024$ & $\mathrm{Br}(3)-\operatorname{Re}(1)-\mathrm{N}(10) 95.685$ \\
$\mathrm{C} 1(3)-\mathrm{TC}(1)-\mathrm{C}(16) 171.045$ & $\mathrm{Br}(3)-\operatorname{Re}(1)-\mathrm{C}(16) 171.151$ \\
$\mathrm{C} 1(3)-\mathrm{TC}(1)-\mathrm{C}(17) 87.930$ & $\mathrm{Br}(3)-\operatorname{Re}(1)-\mathrm{C}(17) 90.133$ \\
$\mathrm{C} 1(4)-\mathrm{TC}(1)-\mathrm{Cl}(6) 89.184$ & $\mathrm{Br}(4)-\operatorname{Re}(1)-\mathrm{Br}(6) 88.191$ \\
$\mathrm{C} 1(4)-\mathrm{TC}(1)-\mathrm{N}(10) 177.610$ & $\mathrm{Br}(4)-\operatorname{Re}(1)-\mathrm{N}(10) 175.860$ \\
$\mathrm{C} 1(4)-\mathrm{TC}(1)-\mathrm{C}(16) 88.531$ & $\mathrm{Br}(4)-\operatorname{Re}(1)-\mathrm{C}(16) 88.451$ \\
$\mathrm{C} 1(4)-\mathrm{TC}(1)-\mathrm{C}(17) 91.109$ & $\mathrm{Br}(4)-\operatorname{Re}(1)-\mathrm{C}(17) 84.160$ \\
$\mathrm{C} 1(6)-\mathrm{TC}(1)-\mathrm{N}(10) 88.451$ & $\mathrm{Br}(6)-\operatorname{Re}(1)-\mathrm{N}(10) 95.858$ \\
$\mathrm{C} 1(6)-\mathrm{TC}(1)-\mathrm{C}(16) 92.268$ & $\mathrm{Br}(6)-\operatorname{Re}(1)-\mathrm{C}(16) 87.558$ \\
$\mathrm{C} 1(6)-\mathrm{TC}(1)-\mathrm{C}(17) 179.684$ & $\mathrm{Br}(6)-\operatorname{Re}(1)-\mathrm{C}(17) 172.331$ \\
$\mathrm{~N}(10)-\mathrm{TC}(1)-\mathrm{C}(16) 91.920$ & $\mathrm{~N}(10)-\operatorname{Re}(1)-\mathrm{C}(16) 92.579$ \\
\hline
\end{tabular}




\begin{tabular}{|c|c|c|c|}
\hline $\mathrm{N}(10)-\mathrm{TC}(1)-\mathrm{C}(17) 9$ & 91.257 & $N(10)-\operatorname{Re}(1)-C(17)$ & 91.782 \\
\hline $\mathrm{C}(16)-\mathrm{TC}(1)-\mathrm{C}(17) 8$ & 87.767 & $C(16)-\operatorname{Re}(1)-C(17)$ & 92.766 \\
\hline $\mathrm{Cl}(3)-\mathrm{TC}(2)-\mathrm{Cl}(4) 8$ & 83.569 & $\operatorname{Br}(3)-\operatorname{Re}(2)-\operatorname{Br}(4)$ & 83.667 \\
\hline $\mathrm{Cl}(3)-\mathrm{TC}(2)-\mathrm{Cl}(5) 8$ & 87.085 & $\operatorname{Br}(3)-\operatorname{Re}(2)-\operatorname{Br}(5)$ & 87.629 \\
\hline $\mathrm{Cl}(3)-\mathrm{TC}(2)-\mathrm{C}(9)$ & 170.989 & $\operatorname{Br}(3)-\operatorname{Re}(2)-C(9)$ & 170.658 \\
\hline $\mathrm{Cl}(3)-\mathrm{TC}(2)-\mathrm{C}(12) 8$ & 89.035 & $\operatorname{Br}(3)-\operatorname{Re}(2)-C(12)$ & 89.820 \\
\hline $\mathrm{Cl}(3)-\mathrm{TC}(2)-\mathrm{N}(14) 9$ & 96.842 & $\operatorname{Br}(3)-\operatorname{Re}(2)-\mathrm{N}(14)$ & 96.681 \\
\hline $\mathrm{Cl}(4)-\mathrm{TC}(2)-\mathrm{Cl}(5) 8$ & 88.531 & $\operatorname{Br}(4)-\operatorname{Re}(2)-\operatorname{Br}(5)$ & 88.703 \\
\hline $\mathrm{Cl}(4)-\mathrm{TC}(2)-\mathrm{C}(9) \quad 8$ & 87.995 & $\operatorname{Br}(4)-\operatorname{Re}(2)-C(9)$ & 88.052 \\
\hline $\mathrm{Cl}(4)-\mathrm{TC}(2)-\mathrm{C}(12) 8$ & 87.968 & $\operatorname{Br}(4)-\operatorname{Re}(2)-\mathrm{C}(12)$ & 84.144 \\
\hline $\mathrm{Cl}(4)-\mathrm{TC}(2)-\mathrm{N}(14) 1$ & 177.315 & $\operatorname{Br}(4)-\operatorname{Re}(2)-\mathrm{N}(14)$ & 175.085 \\
\hline $\mathrm{Cl}(5)-\mathrm{TC}(2)-\mathrm{C}(9)$ & 89.537 & $\operatorname{Br}(5)-\operatorname{Re}(2)-C(9)$ & 87.869 \\
\hline $\mathrm{Cl}(5)-\mathrm{TC}(2)-\mathrm{C}(12) 1$ & 175.043 & $\operatorname{Br}(5)-\operatorname{Re}(2)-C(12)$ & 172.634 \\
\hline $\mathrm{Cl}(5)-\mathrm{TC}(2)-\mathrm{N}(14) 9$ & 94.146 & $\operatorname{Br}(5)-\operatorname{Re}(2)-\mathrm{N}(14)$ & 96.209 \\
\hline $\mathrm{C}(9)-\mathrm{TC}(2)-\mathrm{C}(12)$ & 93.845 & $C(9)-\operatorname{Re}(2)-C(12)$ & 93.639 \\
\hline $\mathrm{C}(9)-\mathrm{TC}(2)-\mathrm{N}(14)$ & 91.733 & $\mathrm{C}(9)-\operatorname{Re}(2)-\mathrm{N}(14)$ & 91.938 \\
\hline $\mathrm{C}(12)-\mathrm{TC}(2)-\mathrm{N}(14) 8$ & 89.376 & $\mathrm{C}(12)-\operatorname{Re}(2)-\mathrm{N}(14)$ & 90.951 \\
\hline $\mathrm{TC}(1)-\mathrm{Cl}(3)-\mathrm{TC}(2) 9$ & 96.038 & $\operatorname{Re}(1)-\operatorname{Br}(3)-\operatorname{Re}(2)$ & 96.096 \\
\hline $\mathrm{TC}(1)-\mathrm{Cl}(4)-\mathrm{TC}(2) 9$ & 96.617 & $\operatorname{Re}(1)-\operatorname{Br}(4)-\operatorname{Re}(2)$ & 96.372 \\
\hline $\mathrm{TC}(2)-\mathrm{C}(9)-\mathrm{O}(7)$ & 178.999 & $\operatorname{Re}(2)-C(9)-O(7)$ & 180.000 \\
\hline $\mathrm{TC}(1)-\mathrm{N}(10)-\mathrm{O}(8)$ & 178.950 & $\operatorname{Re}(1)-N(10)-O(8)$ & 178.815 \\
\hline $\operatorname{TC}(2)-C(12)-O(13) 1$ & 172.826 & $\operatorname{Re}(2)-C(12)-O(13)$ & 176.867 \\
\hline $\mathrm{TC}(2)-\mathrm{N}(14)-\mathrm{O}(11) 1$ & 177.332 & $\operatorname{Re}(2)-N(14)-O(11)$ & 178.182 \\
\hline $\mathrm{TC}(1)-\mathrm{C}(16)-\mathrm{O}(15) 1$ & 178.815 & $\operatorname{Re}(1)-C(16)-O(15)$ & 179.368 \\
\hline $\mathrm{TC}(1)-\mathrm{C}(17)-\mathrm{O}(18) 1$ & 179.051 & $\operatorname{Re}(1)-C(17)-O(18)$ & 176.883 \\
\hline
\end{tabular}




\section{HPLC traces:}

HPLC chromatograms of compounds $\left.\left[{ }^{99 \mathrm{~m}} \mathrm{Tc}\left(\mathrm{OH}_{2}\right)_{3}(\mathrm{CO})_{3}\right]^{+},{ }^{99 \mathrm{~m}} \mathrm{TcCl}\left(\mathrm{OH}_{2}\right)_{2}(\mathrm{CO})_{2} \mathrm{NO}\right]^{+}$ $\left.{ }^{99 \mathrm{~m}} \mathrm{TcO}_{4}\right]^{-}$(all gamma traces) and $\left[\operatorname{ReBr}\left(\mathrm{OH}_{2}\right)_{2}(\mathrm{CO})_{2} \mathrm{NO}\right]^{+}(\mathrm{UV}$-trace; $254 \mathrm{~nm})$.
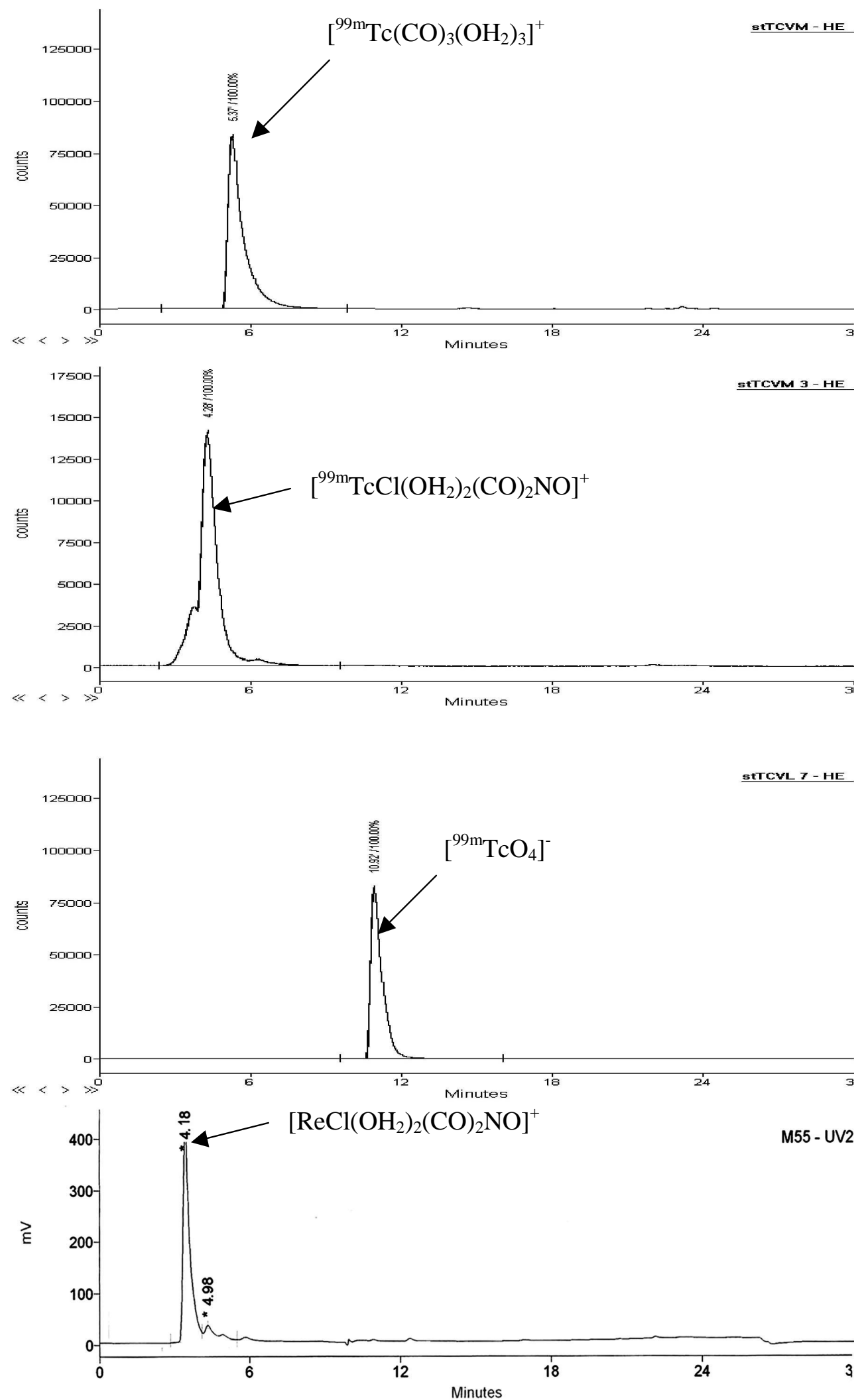


\section{TCL traces}

TLC chromatograms of compounds $\left[{ }^{99 \mathrm{~m}} \mathrm{TcCl}\left(\mathrm{OH}_{2}\right)_{2}(\mathrm{CO})_{2} \mathrm{NO}^{+}\right.$, 2a and $\left[\mathrm{TcO}_{4}\right]^{-}$.

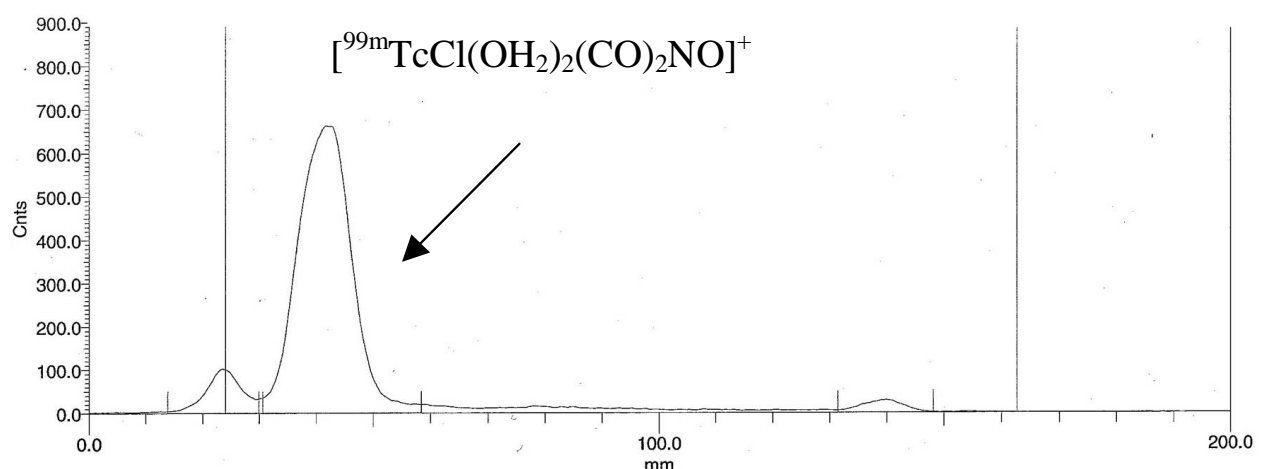

\begin{tabular}{r|c|c|c|c|c|r|r|r|r|r}
$\mathrm{Nr}$ & Name & $\begin{array}{c}\text { Max.Pos } \\
\mathrm{mm}\end{array}$ & $\begin{array}{c}\text { Pos } \\
\mathrm{mm}-\mathrm{mm}\end{array}$ & If_center & if_max & $\begin{array}{c}\text { Breite } \\
\mathrm{mm}\end{array}$ & $\begin{array}{c}\text { Max } \\
\text { Cnts }\end{array}$ & $\begin{array}{c}\text { I } \\
\text { Cnts }\end{array}$ & $\begin{array}{r}1 / \text { Area } \\
\text { Cnts/mm }\end{array}$ & $\begin{array}{c}\% \text { \% } \\
\%\end{array}$ \\
\hline 1 & & 23.3 & $13.8-29.9$ & -0.02 & 0.00 & 16.1 & 102.20 & 3725.1 & 231.4 & 9.11 \\
\hline 2 & none & 41.8 & $30.6-58.4$ & 0.15 & 0.13 & 27.8 & 662.73 & 35913.5 & 1291.9 & 87.84 \\
\hline 3 & none & 139.9 & $131.4-148.1$ & 0.84 & 0.84 & 16.7 & 29.07 & 1248.5 & 74.8 & 3.05 \\
\hline & Total & & & & & 209.3 & & 44731.1 & 213.7 & 109.40 \\
\hline & Sum & & & & & 60.6 & & 40887.1 & 674.7 & 100.00 \\
\hline & Rem & & & & & 148.7 & & 3844.1 & 25.8 & 9.40 \\
\hline
\end{tabular}
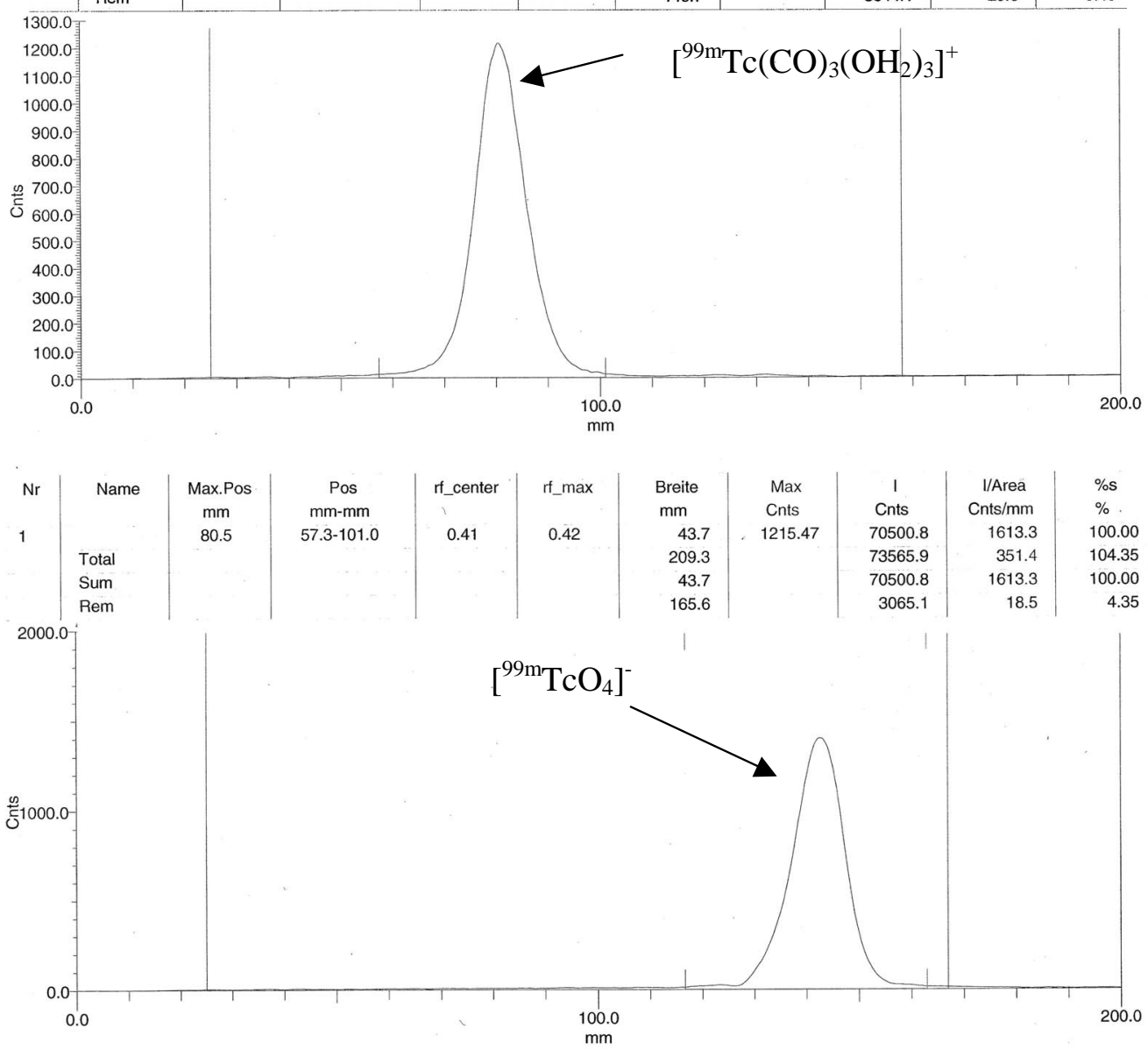

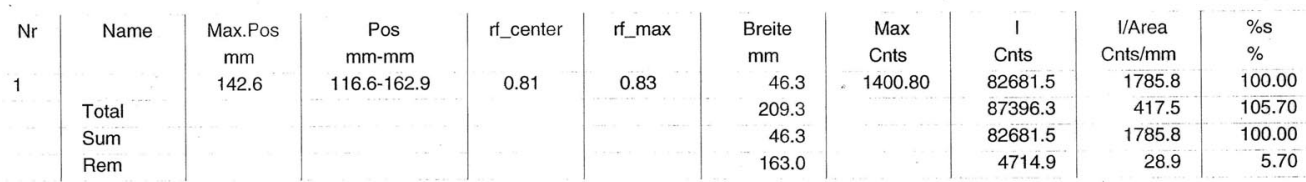

